\title{
Fundamenta Morphologicae Mathematicae *
}

\author{
John Goutsias $\dagger$ \\ Center for Imaging Science and \\ Department of Electrical and Computer Engineering \\ The Johns Hopkins University \\ Baltimore, MD 21218, U. S. A. \\ goutsias@mycenae.ece.jhu.edu
}

Henk J.A.M. Heijmans

Centre for Mathematics and Computer Science

P.O. Box 94079

1090 GB Amsterdam, The Netherlands

Henk.Heijmans@cwi.nl

\begin{abstract}
Mathematical morphology is a geometric approach in image processing and analysis with a strong mathematical flavor. Originally, it was developed as a powerful tool for shape analysis in binary and, later, grey-scale images. But it was soon recognized that the underlying ideas could be extended naturally to a much wider class of mathematical objects, namely complete lattices. This paper presents, in a bird's eye view, the foundations of mathematical morphology, or more precisely, the theory of morphological operators on complete lattices.
\end{abstract}

Keywords: adjunction, alternating sequential filter, Boolean function, closing, complete lattice, connectivity, connected operator, dilation, erosion, flat operator, fuzzy set, grain operator, granulometry, grey-scale image, idempotence, mathematical morphology, morphological filter, morphological operator, multivalued image, opening, thresholding.

\section{Introduction}

In 1975, a seminal book by Georges Matheron, entitled Random Sets and Integral Geometry, appeared in the literature [20]. This book laid down the foundations of a novel technique for

* This work was supported in part by NATO Collaborative Research Grant CRG.971503. The work of the first author was also supported by the Office of Naval Research, Mathematical, Computer, and Information Sciences Division, under ONR Grant N00014-90-1345, and by the National Science Foundation, under NSF Award \#9729576.

${ }^{\dagger}$ Address for correspondence: Center for Imaging Science and Department of Electrical and Computer Engineering, The Johns Hopkins University, Baltimore, MD 21218, U. S. A. 
shape processing and analysis known as mathematical morphology. Mathematical morphology was enriched and subsequently popularized by the highly inspiring book Image Analysis and Mathematical Morphology by Jean Serra [24]. Today, mathematical morphology is considered to be a powerful tool for image analysis, in particular for those applications where geometric aspects are relevant. The main idea is to analyze the shape of objects in an image by "probing" the image with a small geometric template (e.g., line segment, disc, square) known as the structuring element. The choice of the appropriate structuring element strongly depends on the particular application at hand. This however should not be viewed as a limitation, since it usually leads to additional flexibility in algorithm design. The reader will find several examples of this flexibility in this paper, as well as in the various other contributions to this special issue.

\section{Morphological Image Operators}

\subsection{Introduction}

The basic problem in mathematical morphology is to design nonlinear operators that extract relevant topological or geometric information from images. This requires development of a mathematical model for images and a rigorous theory that describes fundamental properties of the desirable image operators. For example, let us consider the case of binary (black and white) images. Binary images can be mathematically modeled as subsets of a given space $E$, which, depending on the application at hand, is assumed to possess some additional structure (topological space, metric space, graph, etc.). Thus, the family of binary images is given by $\mathcal{P}(E)$, the subsets of $E$. In this paper, we set $E=\mathbb{R}^{d}$, the $d$-dimensional Euclidean space, in which case $X$ is a continuous binary image, or $E=\mathbb{Z}^{d}$, the $d$-dimensional discrete space, in which case $X$ is a discrete binary image. Fundamental relationships between binary images can be mathematically specified by means of set inclusions, unions, or intersections. For example, the fact that image $X$ is hidden by another image $Y$ can be modeled by set inclusion: $X \subseteq Y$. If we simultaneously consider two images $X, Y$, then what we see is their union $X \cup Y$. The part of an image $Y$ which is not covered by some other image $X$ is their set difference $Y \backslash X=Y \cap X^{\mathrm{c}}$, where $X^{c}$ denotes the set complement of $X$, also called the background of $X$. Some major references in the area of mathematical morphology are [3-6, 8, 10, 16-20,22-25,31-34].

\subsection{Complete lattices}

The previous discussion can be further formalized by noticing that $\mathcal{P}(E)$ is a complete lattice. A complete lattice $\mathcal{L}$ is a nonempty set furnished with a partial order relationship $\leq$ such that every subset $\mathcal{H}$ of $\mathcal{L}$ has a least upper bound $\vee \mathcal{H}$, called supremum, and a greatest lower bound $\wedge \mathcal{H}$, called infimum. Obviously, $\mathcal{P}(E)$ is a complete lattice if we take set inclusion as the partial ordering. In this case, the infimum is given by set intersection and the supremum by set union.

Another example of a complete lattice is given by $\mathcal{L}=\operatorname{Fun}(E, \mathcal{T})$, the set of functions mapping $E$ into a complete lattice $\mathcal{T}$, furnished with a partial order relationship " $F \leq G \Leftrightarrow$ $F(x) \leq G(x)$, for every $x \in E$." Here, the inequality " $F(x) \leq G(x)$ " refers to the partial 
ordering on the complete lattice $\mathcal{T}$, which, in the given context, could be interpreted as the set of "grey-levels." We will encounter different choices of $\mathcal{T}$, such as $\mathcal{T}=\mathbb{R}=\mathbb{R} \cup\{-\infty, \infty\}, \mathcal{T}=$ $\overline{\mathbb{Z}}=\mathbb{Z} \cup\{-\infty, \infty\}$, or $\mathcal{T}=\{0,1, \ldots, N\}$, for some finite $N$ (which are all totally ordered), and $\mathcal{T}=\overline{\mathbb{R}}^{p}$, where $p>1$ is an integer. This latter choice corresponds to the so-called multivalued images; see $\S 4.5$. If $E=\mathbb{R}^{d}$, then $\operatorname{Fun}(E, \mathcal{T})$ models continuous grey-scale images; for $E=\mathbb{Z}^{d}$ it models discrete grey-scale images. Notice that $\operatorname{Fun}(E,\{0,1\})$ is isomorphic to $\mathcal{P}(E)$. Therefore, a binary image can be either viewed as a subset of $E$ or as a function from $E$ into $\{0,1\}$.

From our discussion so far, it is becoming clear that the structure of a complete lattice is the appropriate algebraic framework for a general theory of mathematical morphology. This structure allows us to generalize various concepts of mathematical morphology and apply it to a wide variety of different situations without having to re-derive fundamental results. From an algebraic point of view, mathematical morphology is the study of operators on complete lattices. As we explain in the sequel, these operators are required to satisfy additional properties, such as increasingness, distributivity over suprema or infima, idempotence, etc.

Since the partial order $\leq$ is a fundamental relationship in a complete lattice $\mathcal{L}$, mathematical morphology mostly deals with operators $\psi: \mathcal{L} \rightarrow \mathcal{L}$ which preserve such a relationship, in the sense that, if $X \leq Y$, then $\psi(X) \leq \psi(Y)$. These are called increasing operators. The operator $\psi$ is decreasing if it reverses the ordering. A bijective decreasing operator $\nu: \mathcal{L} \rightarrow \mathcal{L}$ such that $\nu^{2}=\mathrm{id}_{\mathcal{L}}$, where $\mathrm{id}_{\mathcal{L}}$ denotes the identity operator on $\mathcal{L}$, is called a negation. If $\nu$ is a negation, then $X^{*}=\nu(X)$ is called the negative of $X$. On $\mathcal{P}(E)$, the mapping $X \rightarrow X^{c}$ defines a negation, whereas, a negation on $\operatorname{Fun}(E, \overline{\mathbb{R}})$, is the mapping $F \rightarrow-F$. The operator $\psi^{*}: \mathcal{L} \rightarrow \mathcal{L}$, given by $\psi^{*}(X)=\left[\psi\left(X^{*}\right)\right]^{*}$, is the negative operator of $\psi$. It is important to notice that an operator may have several negatives depending on the specific negation. In practice, this does not lead to any confusion. Notice that, if $\psi$ is increasing, then $\psi^{*}$ is increasing too. Finally, the invariance domain of an operator $\psi$, denoted by $\operatorname{Inv}(\psi)$, is defined to be the set of all $X \in \mathcal{P}(E)$ that are invariant under $\psi$; i.e.,

$$
\operatorname{Inv}(\psi)=\{X \in \mathcal{P}(E) \mid \psi(X)=X\}
$$

\subsection{Adjunctions}

Let $\mathcal{L}, \mathcal{M}$ be complete lattices. An operator $\varepsilon: \mathcal{L} \rightarrow \mathcal{M}$ is called an erosion if it distributes over infima, i.e., if $\varepsilon\left(\bigwedge_{i} X_{i}\right)=\bigwedge_{i} \varepsilon\left(X_{i}\right)$, for every collection $\left\{X_{i}\right\}$ of elements in $\mathcal{L}$. An operator $\delta$ : $\mathcal{M} \rightarrow \mathcal{L}$ is called a dilation if it distributes over suprema, i.e., if $\delta\left(\bigvee_{i} Y_{i}\right)=\bigvee_{i} \delta\left(Y_{i}\right)$, for every collection $\left\{Y_{i}\right\}$ of elements in $\mathcal{M}$. Two operators $\varepsilon: \mathcal{L} \rightarrow \mathcal{M}$ and $\delta: \mathcal{M} \rightarrow \mathcal{L}$ are said to form an adjunction between $\mathcal{L}$ and $\mathcal{M}$ if

$$
\delta(Y) \leq X \Longleftrightarrow Y \leq \varepsilon(X), X \in \mathcal{L}, Y \in \mathcal{M}
$$

Given an adjunction $(\varepsilon, \delta)$ between $\mathcal{L}$ and $\mathcal{M}$, then $\varepsilon$ is an erosion and $\delta$ is a dilation. In fact, with every erosion $\varepsilon: \mathcal{L} \rightarrow \mathcal{M}$ one can associate a unique dilation $\delta: \mathcal{M} \rightarrow \mathcal{L}$ such that $(\varepsilon, \delta)$ constitutes an adjunction between $\mathcal{L}$ and $\mathcal{M}$. In this case,

$$
\delta(Y)=\bigwedge\{X \in \mathcal{L} \mid Y \leq \varepsilon(X)\}, Y \in \mathcal{M}
$$


Similarly, with every dilation $\delta: \mathcal{M} \rightarrow \mathcal{L}$, one can associate a unique erosion $\varepsilon: \mathcal{L} \rightarrow \mathcal{M}$ such that $(\varepsilon, \delta)$ constitutes an adjunction. In this case,

$$
\varepsilon(X)=\vee\{Y \in \mathcal{M} \mid \delta(Y) \leq X\}, X \in \mathcal{L} \text {. }
$$

In general, the operators $\varepsilon$ and $\delta$ in an adjunction $(\varepsilon, \delta)$ are not inverses of each other. In fact, it can be easily seen that $\delta \varepsilon \leq \mathrm{id}_{\mathcal{L}}$, whereas $\varepsilon \delta \geq \mathrm{id}_{\mathcal{M}}$. However, if $(\varepsilon, \delta)$ is an adjunction, then $\varepsilon \delta \varepsilon=\varepsilon$ and $\delta \varepsilon \delta=\delta$. A very important result is that, every increasing operator $\psi: \mathcal{L} \rightarrow \mathcal{L}$ with $\psi(I)=I$, where $I$ is the greatest element of $\mathcal{L}$ (i.e., $X \leq I$, for every $X \in \mathcal{L}$ ), can be decomposed as a supremum of erosions on $\mathcal{L}$. Similarly, every increasing operator $\psi: \mathcal{L} \rightarrow \mathcal{L}$ with $\psi(O)=O$, where $O$ is the least element of $\mathcal{L}$ (i.e., $X \geq O$, for every $X \in \mathcal{L}$ ), can be decomposed as an infimum of dilations on $\mathcal{L}$. Therefore, erosions and dilations are elementary operators which can be viewed as the building blocks of more advanced increasing morphological operators.

\subsection{Openings and closings}

An operator $\alpha: \mathcal{L} \rightarrow \mathcal{L}$ is called an opening if it is increasing, anti-extensive (i.e., $\alpha \leq$ id), and idempotent (i.e., $\alpha^{2}=\alpha$ ). An operator $\beta: \mathcal{L} \rightarrow \mathcal{L}$ is called a closing if it increasing, extensive (i.e., $\beta \geq$ id), and idempotent. For an adjunction $(\varepsilon, \delta)$ between two complete lattices $\mathcal{L}$ and $\mathcal{M}$, the composition $\delta \varepsilon$ (i.e., an erosion followed by a dilation) is an opening on $\mathcal{L}$, called adjunctional opening, whereas the composition $\varepsilon \delta$ (i.e., a dilation followed by an erosion) is a closing on $\mathcal{M}$, called an adjunctional closing. Further results on openings and closings can be found in $\S 5.2$.

\section{Set Morphology}

\subsection{Set dilation and erosion}

Assume that $\delta$ is a dilation on $\mathcal{P}(E)$, where $E$ is a nonempty set. For $X \subseteq E$, we can write

$$
\delta(X)=\bigcup_{x \in X} A(x)
$$

where $A(x):=\delta(\{x\}) \subseteq E$, the dilation of the singleton $\{x\}$. Here, we have used the fact that $\delta$ distributes over unions. Every dilation on $\mathcal{P}(E)$ is of the form (1), and the adjoint erosion is given by

$$
\varepsilon(X)=\{h \in E \mid A(h) \subseteq X\}, X \subseteq E .
$$

In the following subsection, we consider the special case when the operators are invariant under translations. In that case, the sets $A(x)$ are translates of a fixed set, called the structuring element, over the vector $x$.

\subsection{Translation invariance}

A class of operators of particular interest in image processing and analysis are the ones that are translation invariant. In order to study this type of operators, we first need to define what we 
mean by "translation invariance" in a complete lattice framework. Let $T$ be an Abelian group of automorphisms $\tau$ on $\mathcal{L}$. An operator $\psi: \mathcal{L} \rightarrow \mathcal{L}$ is called $T$-invariant if

$$
\psi \tau=\tau \psi, \quad \forall \tau \in T
$$

If $(\varepsilon, \delta)$ is an adjunction on $\mathcal{L}$, then $\varepsilon$ is $T$-invariant if and only if $\delta$ is $T$-invariant. In this case, $(\varepsilon, \delta)$ is called a $T$-invariant adjunction. When $\mathcal{L}=\mathcal{P}(E)$, with $E=\mathbb{R}^{d}$ or $\mathbb{Z}^{d}, T$ can be taken to be the Abelian group of all translations on $E$. For $h \in E$, let us define the translation operator $\tau_{h}$ on $\mathcal{P}(E)$ by $\tau_{h}(X)=X_{h}=\{x+h \mid x \in X\}$. Then, an operator $\psi: \mathcal{P}(E) \rightarrow \mathcal{P}(E)$ is $T$-invariant if $\psi \tau_{h}=\tau_{h} \psi$, for every $h \in E$, that is, if $\psi\left(X_{h}\right)=[\psi(X)]_{h}$. Every $T$-invariant erosion on $\mathcal{P}(E)$ is given by

$$
\varepsilon_{A}(X)=X \ominus A=\left\{h \in E \mid A_{h} \subseteq X\right\}
$$

whereas, every $T$-invariant dilation is given by

$$
\delta_{A}(X)=X \oplus A=\bigcup\left\{A_{x} \mid x \in X\right\}=\left\{h \in E \mid \check{A}_{h} \cap X \neq \emptyset\right\}
$$

where $A \in \mathcal{P}(E)$ is referred to as the structuring element and $\breve{A}=\{-a \mid a \in A\}$ is the reflection of $A$ around the origin. Observe that the expressions above are in agreement with those in (1), (2). The pair $\left(\varepsilon_{A}, \delta_{A}\right)$ forms a $T$-invariant (or translation invariant) adjunction on $\mathcal{P}(E)$. It is clear that a $T$-invariant erosion on $\mathcal{P}(E)$ comprises all points $h$ of $E$ for which the structuring element $A_{h}$, located at $h$, fits inside $X$. On the other hand, a $T$-invariant dilation on $\mathcal{P}(E)$ comprises of all points $h$ of $E$ for which the structuring element $\breve{A}_{h}$, located at $h$, hits $X$. Notice that $X \oplus A=\left(X^{c} \ominus \check{A}\right)^{c}$. Therefore, the dilation $X \oplus A$ is the negative operator of erosion. This defines a duality between binary dilations and erosions, in the sense that dilation of a binary image $X$ by a structuring element $A$ can be viewed as the set complement of the erosion of the background $X^{c}$ of $X$ by $\check{A}$. The effects of erosion and dilation on a binary image are depicted in Fig. 1. If $\psi$ is an increasing $T$-invariant operator on $\mathcal{P}(E)$, then
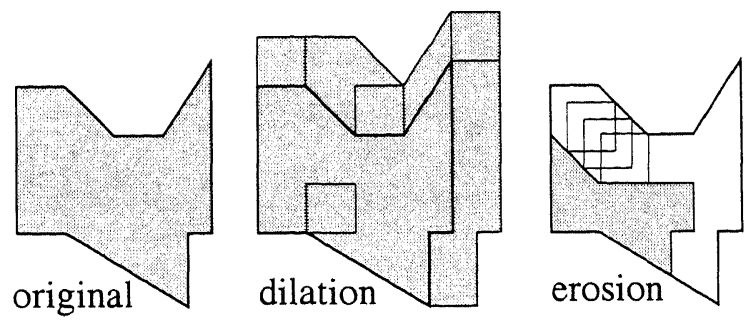

Figure 1 Dilation and erosion by a square structuring element with the origin at the lower-left corner.

$$
\psi(X)=\bigcup_{A \in \mathcal{V}(\psi)} X \ominus A=\bigcap_{A \in \mathcal{V}\left(\psi^{*}\right)} X \oplus \check{A}
$$


Here, $\mathcal{V}(\psi)$ is the kernel of operator $\psi$, defined by $\mathcal{V}(\psi)=\{A \in \mathcal{P}(E) \mid 0 \in \psi(A)\}$, where 0 is the origin in $E$. The result in (5) is known as Matheron's representation theorem.

The decomposition in (5) can be generalized to $T$-invariant operators which are not necessarily increasing. For $A, B \in \mathcal{P}(E)$, the interval $[A, B]$ is the set of all $X \in \mathcal{P}(E)$ with $A \subseteq X \subseteq B$. If $\mathcal{W}(\psi)=\{(A, B) \in \mathcal{P}(E) \times \mathcal{P}(E) \mid[A, B] \subseteq \mathcal{V}(\psi)\}$, and if $\psi$ is a $T$-invariant operator on $\mathcal{P}(E)$, then

$$
\psi(X)=\bigcup_{(A, B) \in \mathcal{W}(\psi)} X \otimes\left(A, B^{c}\right)
$$

where

$$
X \otimes(A, B)=\left\{h \in E \mid A_{h} \subseteq X \text { and } B_{h} \subseteq X^{c}\right\} .
$$

The set $X \otimes(A, B)$ contains all vectors $h$ for which $A_{h}$ does not hit $X^{c}$ and $B_{h}$ does not hit $X$. Notice that $X \otimes(A, B)=(X \ominus A) \cap\left(X^{c} \ominus B\right)$. The mapping $X \mapsto X \otimes(A, B)$ is called the hit-or-miss operator.

Given an adjunction $\left(\varepsilon_{A}, \delta_{A}\right)$ on $\mathcal{P}(E), \delta_{A} \varepsilon_{A}$ defines an opening $\alpha_{A}$ on $\mathcal{P}(E)$, whereas $\varepsilon_{A} \delta_{A}$ defines a closing $\beta_{A}$ :

$$
\begin{aligned}
& \alpha_{A}(X)=X \circ A=(X \ominus A) \oplus A, \\
& \beta_{A}(X)=X \bullet A=(X \oplus A) \ominus A .
\end{aligned}
$$

It is easy to see that the opening $X \circ A$ is the union of all translates of structuring element $A$ that fit inside $X$ :

$$
X \circ A=\bigcup\left\{A_{h} \mid h \in E \text { and } A_{h} \subseteq X\right\} .
$$

Notice that $X \bullet A=\left(X^{c} \circ \check{A}\right)^{c}$. Therefore, the closing $X \bullet A$ is the negative operator of the opening. This defines a duality between openings and closings. The effects of the opening and closing on a binary image are depicted in Fig. 2. Clearly, $X \circ A$ and $X \bullet A$ are smoothing

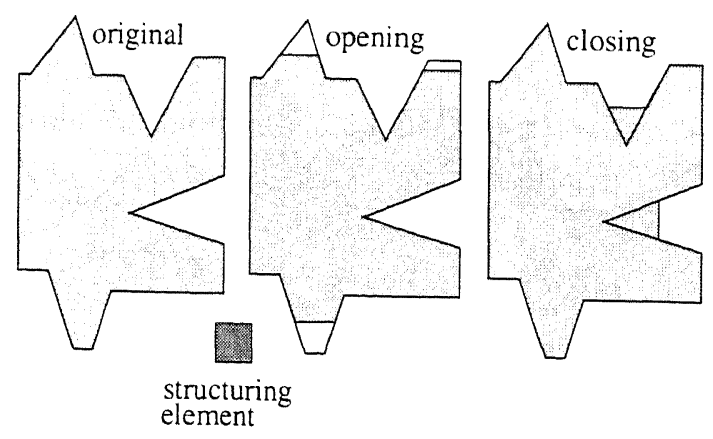

Figure 2. Opening and closing by a square structuring element.

operators that eliminate components in $X$ and $X^{c}$, respectively, of size smaller than the size of the structuring element $A$. 


\subsection{Granulometries}

The notion of a granulometry formalizes the intuitive notion of a sieving process, and happens to be one of the most practical tools in mathematical morphology. Here, we give a brief exposition. More details can be found in [24]. For a formal presentation we refer to [10].

Consider the image space $\mathcal{L}=\mathcal{P}\left(\mathbb{R}^{d}\right)$. For a set $X \subseteq \mathbb{R}^{d}$, denote by $\operatorname{conn}(X)$ the set of connected components of $X$ (see Section 6 for a formal definition of connectivity), also called the particles of $X$. One can think of a sieving process as a parameterized family $\alpha_{r}, r>0$, of operators on $\mathcal{L}$ such that every $\alpha_{r}$ deletes from $X$ all particles "the size of which is at most $r . "$ This last phrase can be formalized in various ways. Thinking of the physical device of a sieve with meshes $r A$, where $A$ is convex-shaped (e.g., $A$ may be a disk or a square in the 2dimensional case), $\alpha_{r}$ deletes from $X$ all particles $Y \in \operatorname{conn}(X)$ which have at least one translate $Y_{h}$ that fits inside $r A$ :

$$
\alpha_{r}(X)=\bigcup\left\{Y \in \operatorname{conn}(X) \mid Y_{h} \nsubseteq r A, \text { for all } h \in \mathbb{R}^{d}\right\} .
$$

It is easy to show (even under the weaker assumption that $A$ is star-shaped with respect to the origin) that the operators $\alpha_{r}$ have the following properties:

(i) $\left\{\alpha_{r} \mid r>0\right\}$ is a family of openings with the semigroup property

$$
\alpha_{r} \alpha_{s}=\alpha_{s} \alpha_{r}=\alpha_{s}, s \geq r
$$

(ii) every $\alpha_{r}$ is translation invariant;

(iii) the family $\alpha_{r}$ is scale-compatible, i.e.,

$$
\alpha_{r}(r X)=r \alpha_{1}(X), \quad r>0, X \subseteq \mathbb{R}^{d} .
$$

A family $\left\{\alpha_{r} \mid r>0\right\}$ of operators on $\mathcal{L}$ that satisfies property $(i)$ is called a granulometry. If it satisfies all three properties $(i)-(i i i)$, then it is called a Minkowski granulometry.

An important instance of a granulometry is the family of openings $\alpha_{r}(X)=X \circ A(r)$, where $\{A(r) \mid r>0\}$ is a one-parameter family of structuring elements with the property that $A(s)$ is $A(r)$-open for $s \geq r$ (a set $A$ is said to be $B$-open if $A \circ B=A$ ). Note that this latter example is completely different from the example we described before.

The next two results yield a complete description of Minkowski granulometries; see [10,20,24]. Proposition 3.1. Every Minkowski granulometry $\left\{\alpha_{r} \mid r>0\right\}$ on $\mathcal{P}\left(\mathbb{R}^{d}\right)$ is of the form

$$
\alpha_{r}(X)=\bigcup_{s \geq r} \bigcup_{A \in \mathcal{A}} X \circ s A
$$

where $\mathcal{A} \subseteq \mathcal{P}(E)$ is an arbitrary collection of structuring elements.

The collection $\mathcal{A}$ can be very huge in practice. For the example described in (6), we find that $\mathcal{A}$ comprises all connected sets $C$ such that $C_{h} \nsubseteq A$, for all $h \in \mathbb{R}^{d}$.

If, in addition, we require that every opening $\alpha_{r}$ is of a structural type, we arrive at the following result.

Proposition 3.2. Given a compact structuring element $A \subseteq \mathbb{R}^{d}$, the openings $\alpha_{r}(X)=X$ or $A$ on $\mathcal{P}\left(\mathbb{R}^{d}\right)$ define a Minkowski granulometry if and only if $A$ is convex. 


\subsection{Boolean functions and morphological operators}

In this subsection, we discuss the relationship between Boolean functions and morphological operators. Our exposition is based on Sect. 4.5 in [10].

First, we recall some basic terminology. A Boolean function (of $n$ variables) is a mapping from the Boolean lattice $\{0,1\}^{n}$ into the Boolean lattice $\{0,1\}$. If $b_{1}, b_{2}$ are Boolean functions, then their product $b_{1} b_{2}$ (infimum) and sum $b_{1}+b_{2}$ (supremum) are Boolean functions as well, given by

$$
\begin{aligned}
\left(b_{1} b_{2}\right)\left(u_{1}, \ldots, u_{n}\right) & =b_{1}\left(u_{1}, \ldots, u_{n}\right) b_{2}\left(u_{1}, \ldots, u_{n}\right), \\
\left(b_{1}+b_{2}\right)\left(u_{1}, \ldots, u_{n}\right) & =b_{1}\left(u_{1}, \ldots, u_{n}\right)+b_{2}\left(u_{1}, \ldots, u_{n}\right) .
\end{aligned}
$$

The complement of a Boolean function $b$ is given by

$$
b^{*}\left(u_{1}, \ldots, u_{n}\right)=1-b\left(u_{1}, \ldots, u_{n}\right) .
$$

The Boolean function $b$ is increasing (or positive) if $u_{i} \leq v_{i}, i=1, \ldots, n$, implies that $b\left(u_{1}, \ldots, u_{n}\right.$ ) $\leq b\left(v_{1}, \ldots, v_{n}\right)$.

For the purpose of this subsection, the class of threshold functions is very important.

Definition 3.1. Given $w_{1}, w_{2}, \ldots, w_{n}$ and $s \in \mathbb{R}$, the Boolean function

$$
b\left(u_{1}, \ldots, u_{n}\right)= \begin{cases}1, & \text { if } \sum_{i=1}^{n} w_{i} u_{i} \geq s \\ 0, & \text { if } \sum_{i=1}^{n} w_{i} u_{i}<s\end{cases}
$$

is called a threshold function. The entries $w_{i}$ are called the weights and $s$ is called the threshold. The vector $\left(w_{1}, \ldots, w_{n} \mid s\right)$ is called the realization vector.

By using the convention that $[S]=1$ if statement $S$ is true and 0 if it is false, we can also write

$$
b\left(u_{1}, \ldots, u_{n}\right)=\left[\sum_{i=1}^{n} w_{i} u_{i} \geq s\right]
$$

An important example is the rank function $r_{s}$ which has realization vector $(1, \ldots, 1 \mid s)$, i.e.,

$$
r_{s}\left(u_{1}, \ldots, u_{n}\right)=\left[\sum_{i=1}^{n} u_{i} \geq s\right] .
$$

In this subsection, we identify a set $X$ by its characteristic function, i.e., we write $X(h)=1$, if $h \in X$, and $X(h)=0$, if $h \notin X$. Assume that $A$ is a structuring element containing $n$ elements $a_{1}, \ldots, a_{n}$, and assume that $b$ is a Boolean function with $n$ variables. Define the translation invariant operator $\psi_{b}$ by

$$
\psi_{b}(X)=\left\{h \in E \mid b\left(X\left(a_{1}+h\right), \ldots, X\left(a_{n}+h\right)\right)=1\right\} .
$$

Notice that, if $b\left(u_{1}, \ldots, u_{n}\right)=u_{1} \cdots u_{n}$, then $\psi_{b}(X)=X \ominus A$, whereas, if $b\left(u_{1}, \ldots, u_{n}\right)=$ $u_{1}+\cdots+u_{n}$, then $\psi_{b}(X)=X \oplus \check{A}$. 
Proposition 3.3. $\psi_{b}$ is increasing if and only if $b$ is increasing.

It is easy to check that

$$
\left(\psi_{b}\right)^{*}=\psi_{b^{*}}
$$

As another example of generating morphological operators by means of Boolean functions, we take $b$ to be the rank function $r_{s}$. The resulting operator, which we denote by $\rho_{A, s}$, is called the rank operator. One finds that $h \in \rho_{A, s}(X)$ if and only if $X \cap A_{h}$ contains at least $s$ points. In particular, $\rho_{A, 1}(X)=X \oplus \check{A}$ and $\rho_{A, n}(X)=X \ominus A$. Furthermore,

$$
\rho_{A, n} \leq \rho_{A, n-1} \leq \cdots \leq \rho_{A, 1}
$$

and

$$
\rho_{A, s}^{*}=\rho_{A, n-s+1} .
$$

Thus, $\rho_{A, s}$ is self-dual if $n$ is odd and $s=\frac{1}{2}(n+1)$. The operator $\rho_{A,(n+1) / 2}$ is called the median operator. Fig. 3 illustrates the use of rank operators for image denoising, and for the case when $A$ is the $3 \times 3$ square structuring element.

We get a much larger class of morphological operators if we choose for $b$ an arbitrary threshold function. In fact, if

$$
b\left(u_{1}, \ldots, u_{n}\right)=\left[\sum_{i=1}^{n} w_{i} u_{i} \geq s\right]
$$

then

$$
\psi_{b}(X)=\left\{h \in E \mid \sum_{i=1}^{n} w_{i} X\left(h+a_{i}\right) \geq s\right\} .
$$

We call this operator the weighted rank operator. Considering $w$ as a function from $E$ into $\mathbb{Z}$ with domain $A$ (and $w\left(a_{i}\right)=w_{i}$ ), we can write

$$
\psi_{b}(X)=X \star_{s} w
$$

where $X \star_{s} w=\left\{x \in E \mid \sum_{h \in E} w(h) X(x+h) \geq s\right\}$. Substituting for $w$ the characteristic function of $A$, we get

$$
\rho_{A, s}(X)=X \star_{s} A .
$$

As a final illustration, we explain how to express the hit-or-miss operator $X \mapsto X \circledast(B, C)$ as a weighted rank operator. Let the weight function $w$ be given by

$$
w(h)=B(h)-C(h),
$$

where $B, C$ are considered as characteristic functions; then,

$$
X \circledast(B, C)=X \star_{m} w
$$

Here $m$ denotes the number of points in $B$. In [10], we explain how to construct modifications of the hit-or-miss operator starting from this representation. 


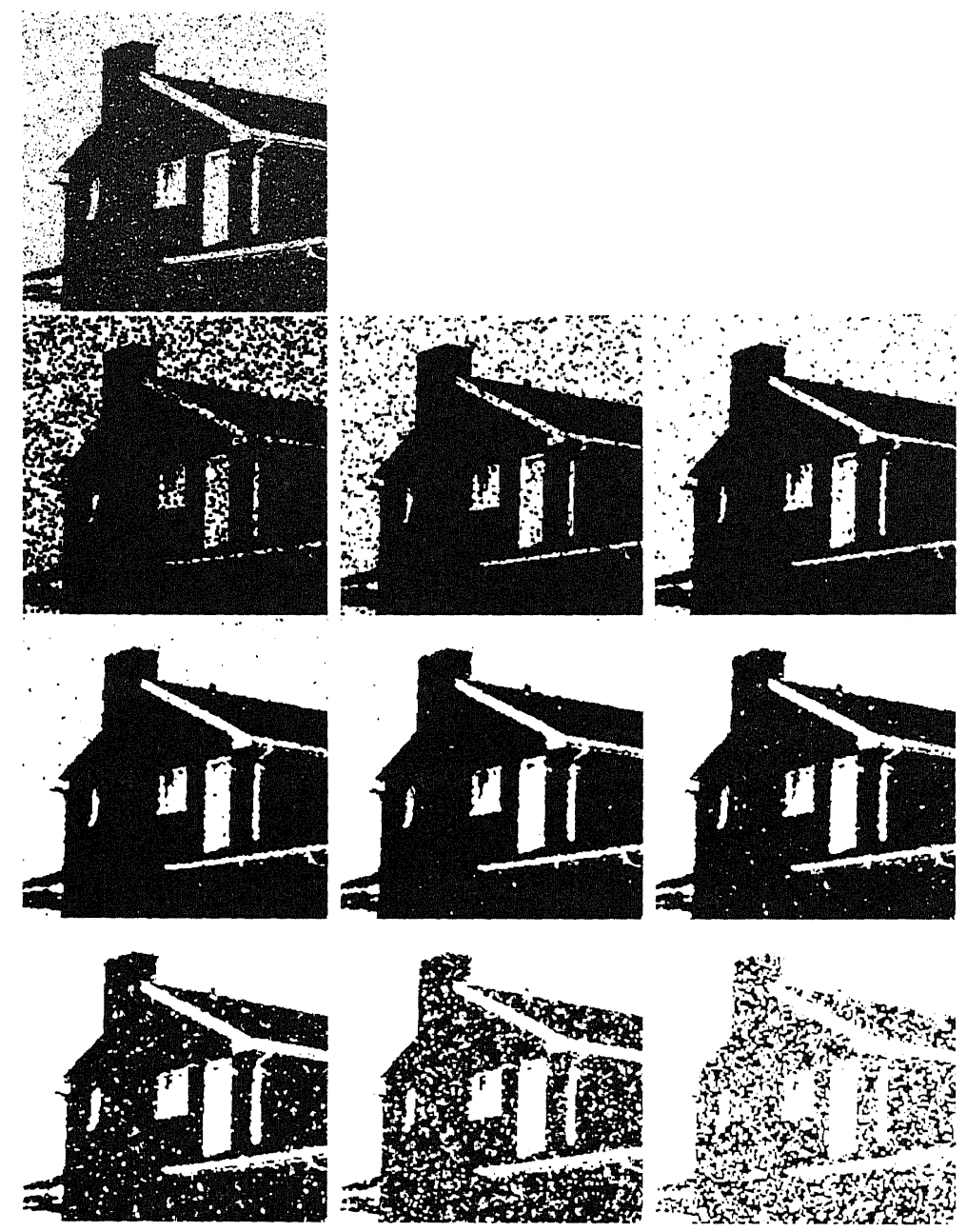

Figure 3 Original noisy image (top) and the transformed images $\rho_{A, n}(X)$, for $n=1, \ldots, 9$ (from left to right and top to bottom), where $A$ is the $3 \times 3$ square structuring element.

We conclude this subsection with the following remarks. The previous exposition is general, in the sense that it applies to the discrete as well as to the continuous case. However, the operators which fall within the class described here use finite structuring elements. As such, this approach is only relevant for the discrete case. Finally, the operators described in this subsection are also known in the literature as stack filters $[19,35]$.

\subsection{Morphology for fuzzy sets}

The expressions for erosion and dilation in (3), (4) can also be written in terms of implications and conjunctions (logical 'AND'):

$$
\varepsilon_{A}(X)=\{h \in E \mid \forall y \in E: y-h \in A \Rightarrow y \in X\},
$$




$$
\delta_{A}(X)=\{h \in E \mid \exists y \in E: h-y \in A \text { and } y \in X\} .
$$

Starting from these expressions, it is straightforward to extend these operations from the complete lattice of sets $\mathcal{P}(E)$ to the complete lattice of fuzzy sets $\overline{\mathcal{P}}(E)$. An element in $\overline{\mathcal{P}}(E)$ can be modeled as a function, the so-called membership function, $X: E \rightarrow[0,1]$. To extend the expressions in $(7),(8)$ to $\overline{\mathcal{P}}(E)$, we need to introduce implications and conjunctions on $[0,1] \times[0,1]$.

Definition 3.2. A mapping $C:[0,1] \times[0,1] \rightarrow[0,1]$ is called a conjunction, if $C(x, y)$ is increasing in both arguments, $C(0,1)=C(1,0)=0$, and $C(1,1)=1$.

Definition 3.3. A mapping $I:[0,1] \times[0,1] \rightarrow[0,1]$ is called an implication, if $I(x, y)$ is decreasing in $x$, increasing in $y, I(0,0)=I(1,1)=1$, and $I(1,0)=0$.

Assume that $I$ is an implication with the additional property that $I(a, \cdot)$ is continuous from the right. Given a fuzzy set $A \in \overline{\mathcal{P}}(E)$, called the fuzzy structuring element, the operator $\varepsilon_{A}$ on $\overline{\mathcal{P}}(E)$, given by

$$
\varepsilon_{A}(X)(h)=\bigwedge_{y \in E} I(A(y-h), X(y))
$$

defines an erosion which is translation invariant. On the other hand, the operator

$$
\delta_{A}(X)(h)=\bigvee_{y \in E} C(A(h-y), X(y))
$$

defines a translation invariant dilation on $\overline{\mathcal{P}}(E)$, if we assume that $C(a, \cdot)$ is continuous from the left. In order that the pair $\left(\varepsilon_{A}, \delta_{A}\right)$, given by $(9),(10)$, forms an adjunction, we have to assume that the pair $(I(a, \cdot), C(a, \cdot))$ is an adjunction on $[0,1]$, for every $a$ in the range of $A$. In other words:

$$
C(a, y) \leq x \quad \Longleftrightarrow \quad y \leq I(a, x)
$$

for $x, y \in[0,1]$ and $a$ in the range of $A$. We present some examples.

Gödel-Brouwer implicator:

$$
\begin{aligned}
& C(a, y)=a \wedge y \\
& I(a, x)= \begin{cases}x, & \text { if } x<a \\
1, & \text { if } x \geq a\end{cases}
\end{aligned}
$$

Lukasiewicz implicator:

$$
\begin{aligned}
C(a, y) & =0 \vee(a+y-1) \\
I(a, x) & =1 \wedge(x-a+1)
\end{aligned}
$$

Kleene-Dienes implicator:

$$
\begin{aligned}
C(a, y) & = \begin{cases}0, & \text { if } y \leq 1-a \\
y, & \text { if } y>1-a\end{cases} \\
I(a, x) & =(1-a) \vee x
\end{aligned}
$$


Reichenbach implicator:

$$
\begin{aligned}
& C(a, y)= \begin{cases}0, & \text { if } y \leq 1-a \\
(y+a-1) / a, & \text { if } y>1-a\end{cases} \\
& I(a, x)=1-a+a x
\end{aligned}
$$

There exist various papers dealing with extensions of mathematical morphology to fuzzy sets; see for example $[1,21,29,30]$. In this subsection, we only wanted to show that the theory of adjunctions on complete lattices is a natural framework for such an extension.

\section{Grey-scale Morphology}

We start with some notation and terminology. Mathematically, we represent grey-scale images as functions $F: E \rightarrow \mathcal{T}$, where $\mathcal{T}$ is the grey-value set. We denote the set of all such functions by $\operatorname{Fun}(E, \mathcal{T})$. Depending on the application at hand, we may choose for $\mathcal{T}$ the sets $\overline{\mathbb{R}}=$ $\mathbb{R} \cup\{-\infty,+\infty\}, \overline{\mathbb{R}}_{+}=\mathbb{R}_{+} \cup\{+\infty\}, \overline{\mathbb{Z}}=\mathbb{Z} \cup\{-\infty,+\infty\}, \overline{\mathbb{Z}}_{+}=\mathbb{Z}_{+} \cup\{+\infty\}$, the bounded interval $[0,1]$, or the finite set $\{0,1, \ldots, N\}$. All these examples have a complete lattice structure in comm...n. If $\mathcal{T}=\overline{\mathbb{R}}$, we simply write Fun $(E)$; in all other cases, we include the grey-value set in our notation. An operator $\Psi$ on $\operatorname{Fun}(E, \mathcal{T})$ will be called a function operator.

\subsection{Direct approach}

We assume throughout this subsection that $\mathcal{T}=\overline{\mathbb{R}}$. However, most of our discussion carries over to the case of $\mathcal{T}=\overline{\mathbb{Z}}$. For $h \in E$ and $F \in \operatorname{Fun}(E)$, the horizontal translate $F_{h}$ is defined by

$$
F_{h}(x)=F(x-h), \quad x \in E .
$$

'The vertical translate $F+v$, where $v \in \mathbb{R}$, is defined by

$$
(F+v)(x)=F(x)+v, \quad x \in E .
$$

Given a function $G \in \operatorname{Fun}(E)$, we define the operators

$$
\Delta_{G}(F)=F \oplus G \text { and } \quad \mathcal{E}_{G}(F)=F \ominus G,
$$

where

$$
\begin{aligned}
& (F \oplus G)(x)=\bigvee_{h \in E}[F(x-h)+G(h)], \\
& (F \ominus G)(x)=\bigwedge_{h \in E}[F(x+h)-G(h)] .
\end{aligned}
$$

These operators are the grey-scale dilation and erosion, respectively. We call $G$ an additive structuring function. In the case of ambiguous expressions, we use the convention that $s+t=$ $-\infty$, if $s=-\infty$ or $t=-\infty$, and $s-t=+\infty$, if $s=+\infty$ or $t=-\infty$. Refer to Fig. 4 for an
illustration. 


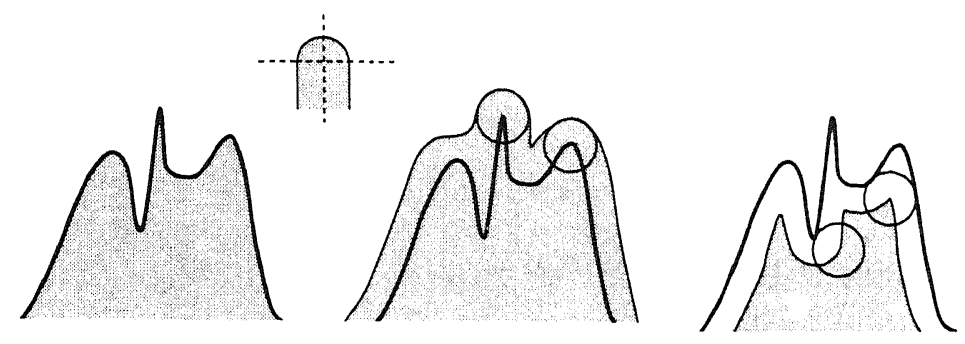

Figure 4. Dilation and erosion of a grey-scale function with an additive strur .ring function.

Proposition 4.1. The pair $\left(\mathcal{E}_{G}, \Delta_{G}\right)$ defines an adjunction on $\operatorname{Fun}(E)$.

Both $\Delta_{G}$ and $\mathcal{E}_{G}$ are translation invariant with respect to horizontal and vertical translations, i.e., both operators have the following property:

$$
\Psi\left(F_{h}+v\right)=[\Psi(F)]_{h}+v
$$

for $h \in E$ and $v \in \mathbb{R}$. An operator with this property is called a T-operator. If $\Psi$ is only invariant under horizontal translations, i.e., if

$$
\Psi\left(F_{h}\right)=[\Psi(F)]_{h},
$$

then it is called an $H$-operator.

The mapping $F \rightarrow-F$, where $(-F)(x)=-F(x)$, defines a negation on $F u n(E)$. Writing $F^{*}=-F$, we have the following duality relations:

$$
(F \oplus G)^{*}=F^{*} \ominus \check{G} \text { and }(F \ominus G)^{*}=F^{*} \oplus \check{G},
$$

where $\check{G}$ is the reflection of $G$ with respect to the origin, that is, $\check{G}(x)=G(-x)$.

There exists a simple analogue of Matheron's representation theorem (see (5)) in the greyscale case. Before we formulate it, we have to generalize the notion of the kernel, given in $\S 3.2$. The kernel of a function operator $\Psi$ is defined by

$$
\mathcal{V}(\Psi)=\{G \in \operatorname{Fun}(E) \mid \Psi(G)(0) \geq 0\} .
$$

Proposition 4.2. Let $\Psi$ be an increasing T-operator on $\operatorname{Fun}(E)$. Then,

$$
\Psi(F)=\bigvee_{G \in \mathcal{V}(\Psi)} F \ominus G=\bigwedge_{G \in \mathcal{V}\left(\Psi^{*}\right)} F \oplus \check{G}
$$

By composing dilations and erosions, we get openings and closings, just like in the binary case.

A general way to construct $T$-operators is by using (extensions of) Boolean functions. If $b$ is an increasing Boolean function of $n$ variables, then we extend $b$ to a function mapping $\overline{\mathbb{R}}^{n}$ into 
$\overline{\mathbb{R}}$ as follows: products are replaced by infima, sums by suprema, 0 by $-\infty$ and 1 by $+\infty$. For example, if $b\left(u_{1}, u_{2}, u_{3}\right)=u_{1}+u_{2} u_{3}$, then $b\left(t_{1}, t_{2}, t_{3}\right)=t_{1} \vee\left(t_{2} \wedge t_{3}\right)$.

Let $A=\left\{a_{1}, \ldots, a_{n}\right\}$ be a finite structuring element and $b$ an increasing Boolean function of $n$ variables. Define the increasing $T$-operator $\Psi_{b}$ on $\operatorname{Fun}(E)$ by

$$
\Psi_{b}(F)(x)=b\left(F\left(x+a_{1}\right), \ldots, F\left(x+a_{n}\right)\right) .
$$

For example, assume that $b$ is a threshold function with realization vector $\left(w_{1}, \ldots, w_{n} \mid s\right)$ containing only integer numbers. Just like in the binary case, let $F \star_{s} w$ denote the transformed function. Then, $\left(F \star_{s} w\right)(x)$ is the $s^{\text {th }}$ entry of the sequence obtained by sorting the values $F\left(x+a_{i}\right)$, occurring $w_{i}$ times, in decreasing order. If every $w_{i}$ equals 1 , we get the rank operator $\rho_{A, s}(F)=F \star_{s} A$. In fact, all grey-scale operators obtained using Boolean functions belong to the class of flat operators introduced in $\S 4.4$.

\subsection{Complete lattice approach}

We have observed that, provided that $\mathcal{T}$ is a complete lattice, $\operatorname{Fun}(E, \mathcal{T})$ is also a complete lattice with partial ordering

$$
F \leq F^{\prime} \quad \text { if } \quad F(x) \leq F^{\prime}(x), \text { for } x \in E .
$$

Heijmans and Ronse [14] (see also [10, Sect. 5.1]) have given a complete description of adjunctions on such complete lattices.

Proposition 4.3. The pair $(\mathcal{E}, \Delta)$ is an adjunction on $\operatorname{Fun}(E, \mathcal{T})$ if and only if, for every $x, y \in E$, there exists an adjunction $\left(e_{y, x}, d_{x, y}\right)$ on $\mathcal{T}$ such that

$$
\begin{aligned}
& \Delta(F)(y)=\bigvee_{x \in E} d_{x, y}(F(x)), \\
& \mathcal{E}(F)(x)=\bigwedge_{y \in E} e_{y, x}(F(y)) .
\end{aligned}
$$

Let us now focus on adjunctions in which both the dilation and the erosion are $H$-operators; such adjunctions are called $H$-adjunctions. The next proposition follows easily from the previous one.

Proposition 4.4. The pair $(\mathcal{E}, \Delta)$ is an $H$-adjunction on $\operatorname{Fun}(E, \mathcal{T})$ if and only if, for every $h \in E$, there exists an adjunction $\left(e_{h}, d_{h}\right)$ on $\mathcal{T}$ such that

$$
\begin{aligned}
& \Delta(F)(y)=\bigvee_{h \in E} d_{h}(F(y-h)), \\
& \mathcal{E}(F)(x)=\bigwedge_{h \in E} e_{h}(F(x+h)) .
\end{aligned}
$$

Assume, for example, that $\mathcal{T}=\overline{\mathbb{R}}$. We obtain the adjunction $\left(\mathcal{E}_{G}, \Delta_{G}\right)$, given by (11)-(13), by setting $d_{h}(t)=t+G(h)$ and $e_{h}(t)=t-G(h)$.

In [10, Sect. 11.5], one finds examples of other $H$-adjunctions. Below, we apply Proposition 4.4 to the case when the grey-value set $\mathcal{T}$ is finite. 


\subsection{Finite grey-value set}

If $\mathcal{T}=\{0,1, \ldots, N\}$, the adjunction in (11)-(13) looses its meaning, since $\mathcal{T}$ is not closed under addition and subtraction. If one tries to overcome this problem, by truncating values below 0 and above $N$, one does not get adjunctions (see [9] or [10, Sect. 11.9]). It turns out that we can use the characterization of $H$-adjunctions, given in Proposition 4.4, to solve this problem.

For $v \in \mathbb{Z}$, define the operation $t \mapsto t \dot{+} v$ on $\{0,1, \ldots, N\}$ as follows:

$$
\begin{cases}0 \dot{+} v=0, & \text { if } t>0 \text { and } t+v \leq 0 \\ t+v=0, & \text { if } t>0 \text { and } 0 \leq t+v \leq N \\ t+v=t+v, & \text { if } t>0 \text { and } t+v>N\end{cases}
$$

Moreover, define the operation $t \mapsto t \dot{-} v$ on $\{0,1, \ldots, N\}$ by

$$
\begin{cases}t \dot{-} v=0, & \text { if } t<N \text { and } t-v \leq 0, \\ t \dot{-} v=t-v, & \text { if } t<N \text { and } 0 \leq t-v \leq N \\ t \dot{-} v=N, & \text { if } t<N \text { and } t-v>N, \\ N \dot{-} v=N . & \end{cases}
$$

Let, for example, $N=10$. Then $(6 \dot{+}) \dot{-} 4=10$ and $6 \dot{+}(5-4)=7$. The operation $\dot{+}$ is neither commutative $(0 \dot{+} \neq 1 \neq 1+0)$ nor associative $((3 \dot{+} 0) \dot{+} 5=3 \dot{+} 5=8 \neq 3=3 \dot{+} 0=3 \dot{+}(0 \dot{+} 5))$.

Lemma 4.1. The pair $e(t)=t \dot{-} v, d(t)=t \dot{+} v$ defines an adjunction on $\{0,1, \ldots, N\}$, for every $v \in \mathbb{Z}$.

For an illustration, see Fig. 5.

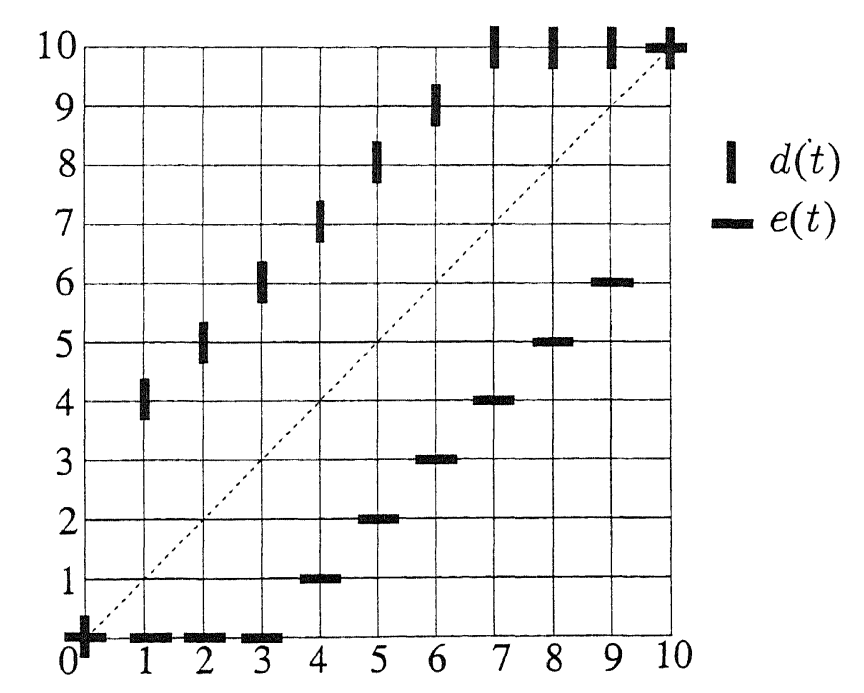

Figure 5. The adjunction $e(t)=t \dot{-} 3, d(t)=t \dot{+} 3$ on $\{0,1, \ldots, 10\}$. 
Combination of Proposition 4.4 and Lemma 4.1 yields an interesting class of $H$-adjunctions, with dilation and erosion given by

$$
\begin{aligned}
(F \dot{\oplus} G)(x) & =\bigvee_{h \in \operatorname{dom}(G)}(F(x-h) \dot{+} G(h)), \\
(F \dot{\theta} G)(x) & =\bigwedge_{h \in \operatorname{dom}(G)}(F(x+h) \dot{-} G(h)),
\end{aligned}
$$

respectively. Here, $G$ is a function with domain $\operatorname{dom}(G)$ and values in $\mathbb{Z}$. In fact, one takes $d_{h}(t)=t \dot{+} G(h), e_{h}(t)=t \dot{-} G(h)$, for $h \in \operatorname{dom}(G)$, and $d_{h} \equiv 0, e_{h} \equiv N$, for $h \notin \operatorname{dom}(G)$. It is easy to verify that

$$
\begin{aligned}
& (F \dot{+} v) \dot{\oplus} G=(F \dot{\oplus} G) \dot{+} v, \\
& (F \dot{-} v) \dot{\theta} G=(F \dot{\theta} G) \dot{-} v,
\end{aligned}
$$

if $v \geq 0$. Additional results can be found in [10, Sect. 11.9].

\subsection{Flat operators and thresholding}

A simple way to construct morphological operators on $\operatorname{Fun}(E)$ is the following: threshold a function at every grey-value, apply a given set operator to the sets obtained in this way, and reconstruct a new transformed function from the transformed sets. This approach can be formalized within the complete lattice framework. Below, we present a short discussion. Further details can be found in [10, Chapters 10-11].

Let $\mathcal{L}$ be an arbitrary complete lattice, let $\mathcal{L} \diamond \overline{\mathbb{R}}$ consist of all functions $\mathbf{X}: \overline{\mathbb{R}} \rightarrow \mathcal{L}$ which are decreasing, and let $\mathcal{L} \Delta \overline{\mathbb{R}}$ be the subcollection of all $\mathbf{X}$ that satisfy

$$
\mathbf{X}\left(\bigvee_{i \in I} t_{i}\right)=\bigwedge_{i \in I} \mathbf{X}\left(t_{i}\right)
$$

for every family $\left\{t_{i} \mid i \in I\right\}$ in $\overline{\mathbb{R}}$. Let $\hat{\mathrm{I}}: \mathcal{L} \diamond \overline{\mathbb{R}} \rightarrow \mathcal{L} \diamond \overline{\mathbb{R}}$ be the operator given by

$$
(\hat{\mathbf{I} X})(t)=\bigwedge_{s<t} \mathbf{X}(s)
$$

Proposition 4.5. The operator $\hat{\mathbf{I}}$ is a closing with invariance domain $\mathcal{L} \Delta \overline{\mathbb{R}}$.

A straightforward consequence of this result is that $\mathcal{L} \Delta \overline{\mathbb{R}}$ is a complete lattice with the same infimum as $\mathcal{L} \diamond \overline{\mathbb{R}}$ (i.e., the pointwise infimum of $\mathcal{L}$ ). The supremum of the family $X_{i}$ in $\mathcal{L} \diamond \overline{\mathbb{R}}$ is given by $\hat{\mathbf{I}}\left(\bigvee_{i \in I} X_{i}\right)$, where the term within parentheses denotes pointwise supremum.

Now, let $F \in \operatorname{Fun}(E)$, and define the threshold sets $\mathbf{X}(F, t)$ by

$$
\mathbf{X}(F, t)=\{x \in E \mid F(x) \geq t\} .
$$

Furthermore, given an element $\mathbf{X} \in \mathcal{P}(E) \triangle \overline{\mathbb{R}}$, define the function

$$
\mathbf{F}(\mathbf{X})(x)=\bigvee\{t \in \overline{\mathbb{R}} \mid x \in \mathbf{X}(t)\}
$$

The next result can be found in [10, Chapter 11]. 
Proposition 4.6. The mapping $F \mapsto \mathbf{X}(F, \cdot)$ defines an isomorphism between Fun $(E)$ and $\mathcal{P}(E) \Delta \overline{\mathbb{R}}$ with inverse $\mathbf{F}$.

Remark: It is possible to use the same approach to deal with u.s.c. (upper semi-continuous) functions, presumed that $E$ is a topological space. In fact, using the same expressions, one can establish an isomorphism between the u.s.c. functions and the complete lattice $\mathcal{F}(E) \triangle \overline{\mathbb{R}}$; here, $\mathcal{F}(E)$ are the closed subsets of $E$.

Using the threshold set representation of $\operatorname{Fun}(E)$, i.e., $\mathcal{P}(E) \triangle \overline{\mathbb{R}}$, one can extend a given increasing set operator $\psi$ on $\mathcal{P}(E)$ to a function operator $\Psi$ on $\operatorname{Fun}(E)$. Let $F$ be a function and apply $\psi$ to every threshold set $\mathbf{X}(F, t)$. The resulting collection of sets $\psi(\mathbf{X}(F, t))$ lies in $\mathcal{P}(E) \diamond \overline{\mathbb{R}}$, but not necessarily in $\mathcal{P}(E) \triangle \overline{\mathbb{R}}$. To achieve this, we apply $\hat{\mathbf{I}}$. Thus, we define

$$
\Psi(F)=\mathbf{F} \hat{\mathbf{I}}(\psi(\mathbf{X}(F, \cdot))) .
$$

It is easy to verify that the following relations hold:

$$
\mathbf{X}(\Psi(F), t)=\bigcap_{s<t} \psi(\mathbf{X}(F, s))
$$

and

$$
\Psi(F)(x)=\bigvee\{t \in \overline{\mathbb{R}} \mid x \in \psi(\mathbf{X}(F, t))\} .
$$

We call $\Psi$ the flat function operator generated by $\psi$. Evidently, $\Psi$ is an increasing operator.

Proposition 4.7. Every increasing flat function operator is invariant under vertical translations. Moreover, if $\psi$ is translation invariant, then $\Psi$ is a $T$-operator.

In $[10$, Sect.11.3], we have derived several interesting results for flat operators. Among others, we showed that $\Psi$ is a dilation if $\psi$ is a dilation; the same holds for erosions, openings, closings, etc.

We conclude with an example. Let $A \subseteq E$ be a structuring element. The flat function operators generated by $\varepsilon_{A}(X)=X \ominus A$ and $\delta_{A}(X)=X \oplus A$ are given by

$$
\mathcal{E}_{A}(F)=\bigwedge_{h \in A} F_{-h} \text { and } \Delta_{A}(F)=\bigvee_{h \in A} F_{h}
$$

respectively. An illustration of flat dilation, erosion, closing, and opening is depicted in Fig. 6 .

\subsection{Multivalued images}

So far, we have been concerned exclusively with binary and grey-scale images. In various applications, however, one is faced with multivalued images. The best known example are color images, but one can also think of the case of magnetic resonance images (MRI) in medical diagnostics; here, a recording usually comprises three images, a proton density image and two relaxation time images. If one wants to analyze such images using morphological tools, one is 

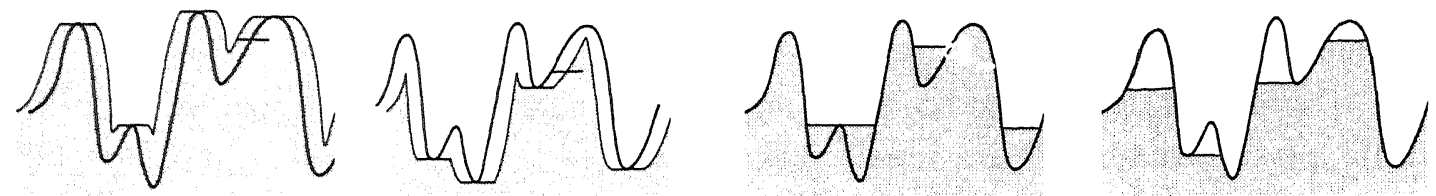

Figure 6. Flat dilation, erosion, closing, and opening.

faced with the problem that their range is no longer a subset of a linearly (or totally) ordered set, but rather a set that is only partially ordered (e.g., $\mathbb{R}^{3}$ in the case of color images).

The fact that images take values in a set which is not totally ordered gives rise to a number of problems. For example, a supremum over a finite subset usually results in a value which does not lie within this finite subset. In color image processing, this gives rise to the so-called "false colors." These, and other problems, are discussed in more detail in [7] and the references mentioned there. In [7], we have discussed, in great detail, a complete lattice approach for multivalued images. Here, we briefly review this approach.

In the sequel, we assume that $\mathcal{R}$ is a complete lattice, that $\mathcal{T}$ is an arbitrary nonempty set, and that $h: \mathcal{T} \rightarrow \mathcal{R}$ is a surjective mapping. Consider a mapping $h^{\leftarrow}: \mathcal{R} \rightarrow \mathcal{T}$ such that $h h^{\leftarrow}(r)=r$, for $r \in \mathcal{R}$. Such a mapping $h^{\leftarrow}$ is called a semi-inverse of $h$. We define a binary relation $\leq_{h}$ on $\mathcal{T}$ in the following way:

$$
t \leq_{h} t^{\prime} \Longleftrightarrow h(t) \leq h\left(t^{\prime}\right), \quad t, t^{\prime} \in \mathcal{T}
$$

Clearly, this relation is reflexive $\left(t \leq_{h} t\right)$ and transitive $\left(t_{1} \leq_{h} t_{2}\right.$ and $t_{2} \leq_{h} t_{3}$ implies that $\left.t_{1} \leq_{h} t_{3}\right)$. However, $\leq_{h}$ does not define a partial ordering because $t \leq_{h} t^{\prime}$ and $t^{\prime} \leq_{h} t$ implies only that $h(t)=h\left(t^{\prime}\right)$ (which we denote as $t={ }_{h} t^{\prime}$ ) but not $t=t^{\prime}$. We refer to $\leq_{h}$ as the $h$-ordering.

A mapping $a: \mathcal{T} \rightarrow \mathcal{T}$ is called $h$-increasing if $t \leq_{h} t^{\prime}$ implies that $a(t) \leq_{h} a\left(t^{\prime}\right)$. One can show that an operator is $h$-increasing if and only if there exists an increasing mapping $a^{\circ}$ : $\mathcal{R} \rightarrow \mathcal{R}$ such that

$$
a^{\circ} h=h a .
$$

The mapping $a^{\circ}$ is uniquely determined by $a$, and can be computed from

$$
a^{\circ}=h a h^{\leftarrow} \text {. }
$$

The mapping $a^{\circ}$ turns-out to be independent of $h^{\leftarrow}$, and we write $a \stackrel{h}{\mapsto} a^{\circ}$.

Definition 4.1. Let $e, d: \mathcal{T} \rightarrow \mathcal{T}$ be two mappings with the property that, for $s, t \in \mathcal{T}$,

$$
d(s) \leq_{h} t \Longleftrightarrow s \leq_{h} e(t) .
$$

Then, the pair $(e, d)$ is called an $h$-adjunction. 
$h$-Adjunctions inherit many properties from classical adjunctions between complete lattices; in particular, the mappings $e$ and $d$ involved are $h$-increasing.

Proposition 4.8. Let $(e, d)$ be $h$-increasing mappings on $\mathcal{T}$, and let $e \stackrel{h}{\mapsto} e^{\circ}, d \stackrel{h}{\mapsto} d^{\circ}$. Then, $(e, d)$ is an $h$-adjunction on $\mathcal{T}$ if and only if $\left(e^{\circ}, d^{\circ}\right)$ is an adjunction on $\mathcal{R}$.

As a next step, we consider adjunctions on $\operatorname{Fun}(E, \mathcal{T})$. Presumed that $h: \mathcal{T} \rightarrow \mathcal{R}$ is surjective, the extension of $h$ to $\operatorname{Fun}(E, \mathcal{T})$, given by

$$
(h(F))(x)=h(F(x)), \quad F \in \operatorname{Fun}(E, \mathcal{T}), x \in E,
$$

is also surjective and possesses (the extension of) $h^{\leftarrow}$ as a semi-inverse.

Proposition 4.9. (a) The pair $(\mathcal{E}, \Delta)$ is an $h$-adjunction on $\operatorname{Fun}(E, \mathcal{T})$ if and only if, for every $x, y \in E$, there exists an $h$-adjunction $\left(e_{x, y}, d_{y, x}\right)$ on $\mathcal{T}$ such that

$$
\begin{aligned}
& h(\mathcal{E}(F)(x))=\bigwedge_{y \in E} h\left(e_{y, x}(F(y))\right), \\
& h(\Delta(F)(x))=\bigvee_{y \in E} h\left(d_{y, x}(F(y))\right),
\end{aligned}
$$

for $F \in \operatorname{Fun}(E, \mathcal{T})$.

(b) Let $e_{x, y} \stackrel{h}{\mapsto} e_{x, y}^{\circ}$ and $d_{y, x} \stackrel{h}{\mapsto} d_{y, x}^{\circ}$. Then, $\left(e_{x, y}^{o}, d_{y, x}^{\circ}\right)$ is an adjunction on $\mathcal{R}$. Let $\left(\mathcal{E}^{\circ}, \Delta^{\circ}\right)$ be the adjunction on $\operatorname{Fun}(E, \mathcal{R})$, given by

$$
\begin{aligned}
\mathcal{E}^{\circ}(F)(x) & =\bigwedge_{y \in E} e_{y, x}^{\circ}(F(y)), \\
\Delta^{\circ}(F)(x) & =\bigvee_{y \in E} d_{y, x}^{o}(F(y)),
\end{aligned}
$$

for $F \in \operatorname{Fun}(E, \mathcal{R})$. Instead of (14), (15), we can write

$$
\begin{aligned}
& h(\mathcal{E}(F)(x))=\mathcal{E}^{\circ}(h(F))(x)=\bigwedge_{y \in E} e_{y, x}^{o}(h(F(y))), \\
& h(\Delta(F)(x))=\Delta^{\circ}(h(F))(x)=\bigvee_{y \in E} d_{y, x}^{\circ}(h(F(y))),
\end{aligned}
$$

for $F \in \operatorname{Fun}(E, \mathcal{T})$. In other words, $\mathcal{E} \stackrel{h}{\mapsto} \mathcal{E}^{\circ}$ and $\Delta \stackrel{h}{\mapsto} \Delta^{\circ}$.

We briefly explain here how the concept of an $h$-adjunction, as well as other derived notions such as $h$-openings and $h$-closings, can be used to deal with spaces of multivalued images, say $\operatorname{Fun}\left(E, \overline{\mathbb{R}}^{p}\right)$. For a comprehensive discussion, we refer to [7]. A possible choice for $h$ is $h(t)=A t$, where $A$ is an invertible $p \times p$ matrix, such as the maximum noise fraction (MNF) transform matrix, used to decorrelate the components of a multispectral image. This choice has been employed in [7] for removing noise from MRI images. Alternatively, we can use a scalar 
transform $h: \overline{\mathbb{R}}^{\mu} \rightarrow \overline{\mathbb{R}}$. For filtering purposes, for example, vector data may be ordered by means of the Mahalanobis distance

$$
h(t)=\left(t-\tau_{0}\right)^{T} C_{0}^{-1}\left(t-\tau_{0}\right),
$$

where $\tau_{0}$ is the expectation of the noise-free image and $C_{0}$ its covariance matrix.

Note that, in Proposition 4.9, the transformed functions $\mathcal{E}(F)$ and $\Delta(F)$ are not uniquely determined, in general. For example, if the function $G$ satisfies the equation in (14), i.e., if $h(G(x))=\bigwedge_{y \in E} h\left(e_{y, x}(F(y))\right)$, then the same holds for $h^{\leftarrow} h(G)$, for every semi-inverse $h^{\leftarrow}$. In [7], we present an algorithm to overcome this problem. The basic idea is to compute $\mathcal{E}(F)(x)$ by substituting the same argument $y$ that yields the infimum in expression (14) (same for $\Delta(F)(x))$.

\section{Morphological Filters}

\subsection{Introduction}

Image filtering is an-important step in image processing and analysis. Filters are used for preprocessing images in order to remove noise and clutter, sharpen edges, and enhance image quality. The theory of morphological filtering was initiated by Matheron and Serra [25] and has found numerous applications in image processing and analysis. In general, a morphological filter (or simply a filter) is an increasing operator $\psi$ on a complete lattice $\mathcal{L}$ that is also idempotent; i.e.,

$$
\psi^{2}=\psi
$$

This property guarantees that repeated application of a filter does not alter the result. In this section, we present a short account on the theory of morphological filters, which is far from being exhaustive. For a more comprehensive discussion, we refer to [12] and the references given here.

\subsection{Openings and closings revisited}

Openings and closings are both increasing and idempotent, and as such they are filters. In fact, an anti-extensive filter is an opening, whereas an extensive filter is a closing. Some preliminary results about openings and closings have been presented in $\S 2.4$. We will continue our discussion about openings and closings here. For simplicity, we restrict ourselves to openings; similar results for closings follow by duality. We start with the following two important results.

Proposition 5.1. Let $\alpha_{1}, \alpha_{2}$ be openings on a complete lattice $\mathcal{L}$. The following assertions are equivalent:

(i) $\alpha_{1} \leq \alpha_{2}$;

(ii) $\quad \alpha_{1} \alpha_{2}=\alpha_{2} \alpha_{1}=\alpha_{1}$;

(iii) $\operatorname{Inv}\left(\alpha_{1}\right) \subseteq \operatorname{Inv}\left(\alpha_{2}\right)$.

In particular, $\alpha_{1}=\alpha_{2}$ if and only if $\operatorname{Inv}\left(\alpha_{1}\right)=\operatorname{Inv}\left(\alpha_{2}\right)$. 
Refer to $[10$, Section 6.1$]$ to see how these relations can be reformulated in terms of adjunctions.

Proposition 5.2. If $\alpha_{i}$ is an opening, for every $i$ in some index set $I$, then $\bigvee_{i \in I} \alpha_{i}$ is an opening as well.

In $\S 2.4$, we have seen that every adjunction $(\varepsilon, \delta)$ between $\mathcal{L}$ and $\mathcal{M}$ yields an opening $\delta \varepsilon$ on $\mathcal{L}$. It can be shown that $\operatorname{Inv}(\delta \varepsilon)=\operatorname{Ran}(\delta)$, where $\operatorname{Ran}(\delta)$ denotes the range of $\delta$. This result can be generalized in the following way.

Proposition 5.3. Let $\alpha$ be an opening on $\mathcal{M}$ and let $(\varepsilon, \delta)$ be an adjunction between $\mathcal{L}$ and $\mathcal{M}$. Then, $\delta \alpha \varepsilon$ is an opening on $\mathcal{L}$ with invariance domain $\{\delta(Y) \mid Y \in \operatorname{Inv}(\alpha)\}$.

\subsection{Adjunctional filters}

If $(\varepsilon, \delta)$ is an adjunction on $\mathcal{L}$, then $\delta^{k} \varepsilon^{k}$ is an opening and $\varepsilon^{k} \delta^{k}$ is a closing, for every $k \geq 1$. This is an immediate consequence of the fact that $\left(\varepsilon^{k}, \delta^{k}\right)$ is an adjunction as well. The composition $\varepsilon \delta^{2} \varepsilon$ is a filter, since $\left(\varepsilon \delta^{2} \varepsilon\right)^{2}=\varepsilon \delta^{2} \varepsilon^{2} \delta^{2} \varepsilon=\varepsilon \delta^{2} \varepsilon$. Here, we have used the fact that $\delta^{2} \varepsilon^{2} \delta^{2}=\delta^{2}$, since $\left(\varepsilon^{2}, \delta^{2}\right)$ is an adjunction. Note also that

$$
\delta \varepsilon \leq \varepsilon \delta^{2} \varepsilon \leq \varepsilon \delta
$$

We state the main result of this subsection.

Proposition 5.4. Assume that $(\varepsilon, \delta)$ is an adjunction on $\mathcal{L}$. Let $\psi$ be a composition of $\varepsilon$ 's and $\delta$ 's of the form

$$
\psi=\varepsilon^{e_{n}} \delta^{d_{n}} \cdots \varepsilon^{e_{2}} \delta^{d_{2}} \varepsilon^{e_{1}} \delta^{d_{1}}
$$

where $e_{i}$ and $d_{i}$ are nonnegative integers. If

$$
\sum_{i=1}^{n} e_{i}=\sum_{i=1}^{n} d_{i}
$$

then $\psi$ is a filter.

Filters of the form (16), which are composed of an equal number of dilations and erosions from an adjunction, are called adjunctional filters. The operator $\varepsilon \delta^{2} \varepsilon$, considered above, is such a filter, but note that it is neither an opening nor a closing; in fact, it is a composition of the closing $\varepsilon \delta$ with the opening $\delta \varepsilon$. In many cases, expressions of the form (16) can be simplified. For example, an expression like $\delta^{4} \varepsilon^{3} \delta^{3}$ may be replaced by $\delta^{4}$, since $\left(\varepsilon^{3}, \delta^{3}\right)$ is an adjunction and, therefore, $\delta^{3} \varepsilon^{3} \delta^{3}=\delta^{3}$, yielding that $\delta^{4} \varepsilon^{3} \delta^{3}=\delta \delta^{3} \varepsilon^{3} \delta^{3}=\delta \delta^{3}=\delta^{4}$. More generally, if a composition contains an expression $\delta^{d^{\prime}} \varepsilon^{e} \delta^{d}$, with $e \leq d$ and $e \leq d^{\prime}$, this can be simplified to $\delta^{d+d^{\prime}-e}$. Dually, $\varepsilon^{e^{\prime}} \delta^{d} \varepsilon^{e}$, with $d \leq e$ and $d \leq e^{\prime}$, can be simplified to $\varepsilon^{e+e^{\prime}-d}$. 


\subsection{Overfilters and inf-overfilters}

If $\psi, \phi$ are two filters, then $\phi \psi, \psi \phi, \phi \vee \psi$ and $\phi \wedge \psi$ are not necessarily filters. In the case of $\phi \vee \psi$, it can be shown that $(\phi \vee \psi)^{2} \geq \phi \vee \psi$, which shows that operator $\phi \vee \psi$ preserves one part of idempotence. Anticipating on the following definition, we say that $\phi \vee \psi$ is an overfilter. Definition 5.1. An increasing operator $\psi$ on a complete lattice is called an overfilter if $\psi^{2} \geq \psi$. It is called an inf-overfilter if $\psi($ id $\wedge \psi)=\psi$.

The dual notions are called underfilters and sup-underfilters, respectively. Motivated by duality, we restrict ourselves to (inf-) overfilters. In the following proposition, we summaries some basic facts concerning these two concepts.

\section{Proposition 5.5.}

(a) Every extensive operator is an inf-overfilter.

(b) Every inf-overfilter is an overfilter.

(c) If $\psi$ is an inf-overfilter, then id $\wedge \psi$ is an opening.

(d) The family of overfilters, as well as the family of inf-overfilters, is closed under suprema.

(e) If $\psi$ is an (inf-) overfilter, then $\psi^{n}$ is an (inf-) overfilter as well, for every nonnegative integer.

There exist various ways to construct (inf-) overfilters. For the sake of illustration, we mention a few of them.

\section{Proposition 5.6.}

(a) Let $(\varepsilon, \delta)$ be an adjunction between $\mathcal{L}$ and $\mathcal{M}$ and let $\psi: \mathcal{M} \rightarrow \mathcal{L}$ be an increasing operator such that $\psi \geq \delta$. Then, $\psi \varepsilon$ is an inf-overfilter.

(b) Let $(\varepsilon, \delta)$ and $\left(\varepsilon^{\prime}, \delta^{\prime}\right)$ be adjunctions between $\mathcal{L}$ and $\mathcal{M}$ such that $\varepsilon^{\prime} \leq \varepsilon$ and (hence) $\delta^{\prime} \geq \delta$. If $\psi$ is an (inf-) overfilter on $\mathcal{M}$, then $\delta^{\prime} \psi \varepsilon$ is an (inf-) overfilter on $\mathcal{L}$.

(c) If $\psi$ is an inf-overfilter and $\beta$ is a closing, then $\beta \psi$ and $\psi \beta \psi$ are inf-overfilters.

We conclude the present subsection with an example. Let $A$ be a finite structuring element containing $n$ points, and let $\mathcal{B}_{k}$ contain all subsets of $A$ which contain $k$ points (where $1 \leq k \leq n$ ). From Proposition 5.6(a), we get that $\delta_{A} \varepsilon_{B}$ is an inf-overfilter, for every structuring element $B \in \mathcal{B}_{k}$. Since the supremum of every collection of inf-overfilters is an inf-overfilter, we get that

$$
\psi_{k}:=\bigvee_{B \in \mathcal{B}_{k}} \delta_{A} \varepsilon_{B}=\delta_{A} \bigvee_{B \in \mathcal{B}_{k}} \varepsilon_{B}
$$

is an inf-overfilter as well. In fact, it is not difficult to verify that $\bigvee_{B \in \mathcal{B}_{k}} \varepsilon_{B}=\rho_{A, k}$, the rank operator introduced in $\S 3.4$. Thus, we have $\psi_{k}=\delta_{A} \rho_{A, k}$. Now, by Proposition $5.5(\mathrm{c})$,

$$
\alpha_{A, k}=\mathrm{id} \wedge \psi_{k}=\mathrm{id} \wedge \delta_{A} \rho_{A, k}
$$

is an opening, called the rank-max opening. For $k=n$, this opening coincides with the structural opening $X \circ A$, whereas for $k=1$ it is the identity operator.

The rank-max opening $\alpha_{A, k}$ is "more tolerant" than the structural opening $\alpha_{A}$. The structural opening preserves translates of $A$ that fit entirely inside $X$, whereas the $\operatorname{rank}$-max opening $\rho_{A, k}$ preserves those portions of $X \cap A_{h}$ that contain at least $k$ points. It is evident that

$$
\alpha_{A, n} \leq \alpha_{A, n-1} \leq \cdots \leq \alpha_{A, 1}=\text { id }
$$




\subsection{Alternating sequential filters}

In this section, we introduce a general class of filters, called alternating sequential filters, or $A S$-filters, obtained by concatenation of overfilters and underfilters. We introduce the following notation: if $\psi_{1}, \psi_{2}, \ldots$ are operators, then

$$
(\psi)_{n}=\psi_{n} \psi_{n-1} \cdots \psi_{1}, \quad n \geq 1
$$

More generally, if $\phi_{1}, \phi_{2}, \ldots$ is another sequence of operators, then

$$
\begin{aligned}
(\psi \phi)_{n} & =\psi_{n} \phi_{n} \psi_{n-1} \phi_{n-1} \cdots \psi_{1} \phi_{1} \\
(\psi \phi \psi)_{n} & =\psi_{n} \phi_{n} \psi_{n} \psi_{n-1} \phi_{n-1} \psi_{n-1} \cdots \psi_{1} \phi_{1} \psi_{1}
\end{aligned}
$$

A sequence of operators $\psi_{1}, \psi_{2}, \ldots$ is said to be absorbing if

$$
\psi_{n} \psi_{m}=\psi_{n}, \quad n \geq m
$$

Proposition 5.7. Given a family of increasing operators $\psi_{n}$, for which $(\psi)_{n}$ is a filter, then the sequence $(\psi)_{n}$ is absorbing.

Proposition 5.8. Assume that $\phi_{1}, \phi_{2}, \ldots$ are overfilters, that $\psi_{1}, \psi_{2}, \ldots$ are underfilters, and that the following conditions are satisfied:

$$
\begin{aligned}
& \phi_{n} \leq \psi_{n}, \\
& \phi_{n} \phi_{n-1} \geq \phi_{n}, \\
& \psi_{n} \psi_{n-1} \leq \psi_{n} .
\end{aligned}
$$

Then, $(\phi \psi)_{n},(\psi \phi)_{n},(\phi \psi \phi)_{n},(\psi \phi \psi)_{n}$ are absorbing filters, and

$$
(\phi \psi \phi)_{n} \leq\left\{\begin{array}{c}
(\phi \psi)_{n} \\
(\psi \phi)_{n}
\end{array}\right\} \leq(\psi \phi \psi)_{n} .
$$

Note that conditions (17)-(19) hold if we make the (stronger) assumption that

$$
\cdots \leq \phi_{3} \leq \phi_{2} \leq \phi_{1} \leq \psi_{1} \leq \psi_{2} \leq \psi_{3} \leq \cdots
$$

The filters constructed in (20) are called alternating sequential filters, or $A S$-filters. The bestknown examples are the ones when $\phi_{n}$ are openings, with $\phi_{n+1} \leq \phi_{n}$, and $\psi_{n}$ are closings, with $\psi_{n+1} \geq \psi_{n}$. In Fig. 7, we depict the effect of three different AS-filters (with increasing parameter $n$ ) on a noisy binary image (top row). The second row depicts the classical AS-filter $(\beta \alpha)_{n}$, for $n=1,2,3$, where $\alpha_{n}(X)=X \circ n A, \beta_{n}(X)=X \bullet n A$, and $A$ is the $3 \times 3$ square structuring element. Here, $n A$ denotes the structuring element $A \oplus A \oplus \cdots \oplus A$ ( $n$ terms). The third row depicts the rank-based AS-filter $\left(\beta_{A} \alpha_{A}\right)_{n}$, for $n=3,6,9$. Here, $\alpha_{A, n}$ is the rank-max opening discussed in $\S 5.4$, and $\beta_{A, n}$ is the negative closing. 

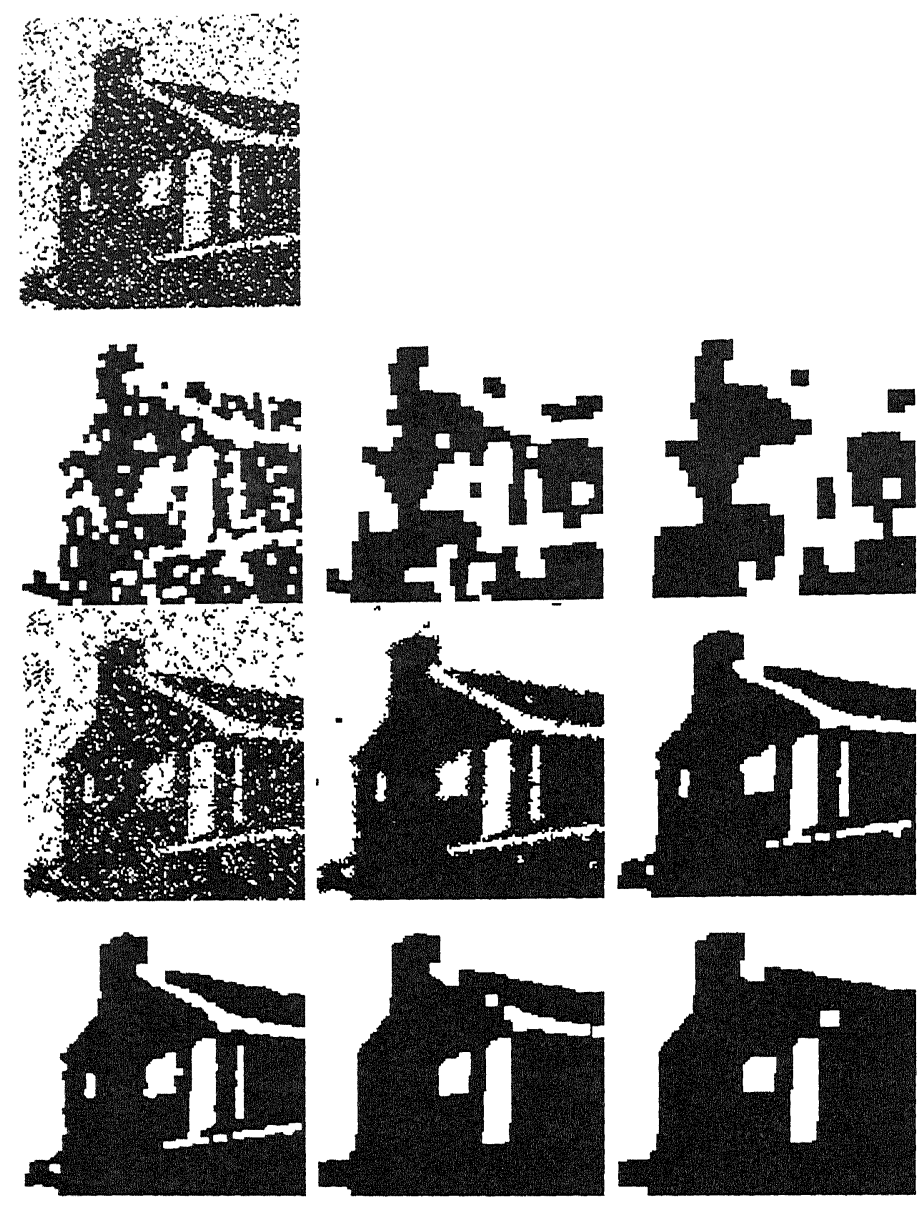

Figure 7 A noisy binary image (top row) and the output resulting from three different AS-filters (three subsequent rows), with increasing parameter $n$ (from left to right). Refer to the text for further details.

As another application, consider the case when $\alpha_{n}, \beta_{n}$ are openings and closings, respectively, and $\xi$ is an increasing operator, such that

$$
\cdots \leq \alpha_{2} \leq \alpha_{1} \leq \xi \leq \beta_{1} \leq \beta_{2} \leq \cdots
$$

Define $\phi_{n}=\alpha_{n} \xi$ and $\psi_{n}=\beta_{n} \xi$. Then, $\phi_{n}$ is an overfilter and, dually, $\psi_{n}$ is an underfilter. It is obvious that (21) holds. As an example, we take for $\xi$ the median operator, using the rhombus mask (origin and four horizontal and vertical neighbors). Let $\alpha_{n}, \beta_{n}$ be the opening and closing, respectively, with the $(2 n+1) \times(2 n+1)$ square structuring element, and define $\phi_{n}=\alpha_{n} \xi$ and $\psi_{n}=\beta_{n} \xi$. It is easy to see that the conditions in (22) are satisfied. The resulting AS-filter $(\psi \phi)_{n}, n=1,2,3$, is illustrated in the bottom row of Fig. 7. 


\subsection{Further results}

Another general method for constructing filters is to iterate increasing operators, which are not idempotent, until convergence. In [15], this method is explained in great detail.

Self-duality is very important in applications: self-dual filters treat image foreground and background identically. In [11], we present a method, based on iteration, to construct self-dual morphological filters.

\section{Connected Operators}

\subsection{Connectivity}

Given a topological space $E$, a set $X \subseteq E$ is said to be connected (or, more precisely, pathconnected) if, for every pair of points $x, y \in X$, there exists a continuous mapping $f$ from $[0,1]$ into $X$ such that $f(0)=x$ and $f(1)=y$. If $X, A$ are two path-connected sets, then $X \oplus A$ is also path-connected. However, this statement is not true for erosions, openings and closings [10]. It is easy to see that the union of a collection of connected sets with nonempty intersection is also connected; i.e., if $\left\{C_{i} \mid i \in I\right\}$ is a collection of connected sets such that $\cap_{i \in I} C_{i} \neq \emptyset$, then $\cup_{i \in I} C_{i}$ is also connected. Matheron and Serra [25] have used this property to generalize the notion of connectivity to spaces $E$ that are not necessarily topological. In this subsection, we give a brief summary of their approach.

Definition 6.1. Let $E$ be an arbitrary nonempty set. A family $\mathcal{C} \subseteq \mathcal{P}(E)$ is called a connectivity class if it satisfies: (i) $\emptyset \in \mathcal{C}$ and $\{x\} \in \mathcal{C}$, for $x \in E$, and (ii) if $C_{i} \in \mathcal{C}$ and $\cap_{i \in I} C_{i} \neq \emptyset$, then $\cup_{i \in I} C_{i} \in \mathcal{C}$. We say that $\mathcal{C}$ defines a connectivity on $E$. An element of $\mathcal{C}$ is called a connected set.

An example, illustrating the notion of connectivity class, is the family $\mathcal{C} \subseteq \mathcal{P}\left(\mathbb{R}^{2}\right)$ comprising all sets whose points cannot be separated by a straight line. It is not difficult to show that $\mathcal{C}$ is indeed a connectivity class. However, elements in $\mathcal{C}$ are not necessarily connected, or path-connected, in the topological sense of connectivity.

Given a connectivity class $\mathcal{C}$, we can define an operator $\gamma_{x}: \mathcal{P}(E) \rightarrow \mathcal{P}(E)$, by

$$
\gamma_{x}(X)=\bigcup\{C \in \mathcal{C} \mid x \in C \text { and } C \subseteq X\} .
$$

Notice that $\gamma_{x}(X) \in \mathcal{C}$ and, therefore, $\gamma_{x}(X)$ is a connected component of $X$ that contains point $x$. It can be shown $[10,25]$ that: $(i) \gamma_{x}$ is an opening on $\mathcal{P}(E)$, known as the connectivity opening associated with $\mathcal{C},(i i) \gamma_{x}(\{x\})=\{x\},($ iii $) \gamma_{x}(X) \cap \gamma_{y}(X)=\emptyset$, or $\gamma_{x}(X)=\gamma_{y}(X)$, and (iv) $x \notin X \Rightarrow \gamma_{x}(X)=\emptyset$. The connected components $\gamma_{x}(X)$ of a set $X$ are called the grains of $X$. Every set $X \subseteq E$ can be uniquely decomposed into its grains $\gamma_{x}(X), x \in E$; i.e.,

$$
X=\bigcup_{x \in E} \gamma_{x}(X)
$$


It can also be shown that, if $\left\{\gamma_{x} \mid x \in E\right\}$ is a collection of operators that satisfy properties (i)-(iv) above, and if $\mathcal{C}$ is given by

$$
\mathcal{C}=\bigcup_{x \in E} \operatorname{Inv}\left(\gamma_{x}\right)
$$

where $\operatorname{Inv}\left(\gamma_{x}\right)$ is the invariance domain of $\gamma_{x}$, then $\mathcal{C}$ defines a connectivity and $\gamma_{x}$ is given by (23). Finally, if $C \subseteq X$ is a connected subset of $X$, then $C$ is contained within some grain of $X$.

Given a connectivity $\mathcal{C}$, we may want to build a new connectivity $\mathcal{C}^{\prime}$ that is more appropriate for a particular application. This can be accomplished by several methods [13]. However, the most frequently used method is to extend $\mathcal{C}$ to $\mathcal{C}^{\prime}$ by means of an increasing operator $\psi$. Indeed, let $\mathcal{C}$ be a connectivity class in $\mathcal{P}(E)$ and let $\psi$ be an increasing operator on $\mathcal{P}(E)$. Let $\mathcal{C}^{\prime}$ consist of the empty set, the singletons, as well as every element $C \in \mathcal{C}$ for which $C \subseteq \psi(C)$; then, $\mathcal{C}^{\prime}$ is a connectivity class [13].

Another way to build a new connectivity from an existing one is by means of dilation. Let $\mathcal{C}$ be a connectivity class in $\mathcal{P}(E)$ with connectivity openings $\gamma_{x}$. Assume that $\delta$ is an extensive dilation on $\mathcal{P}(E)$ such that $\delta(\{x\}) \in \mathcal{C}$, for every $x \in E$. Then,

$$
\mathcal{C}^{\delta}=\{X \subseteq E \mid \delta(X) \in \mathcal{C}\}
$$

is a connectivity class, and $\mathcal{C} \subseteq \mathcal{C}^{\delta}$. The corresponding connectivity openings $\gamma_{x}^{\delta}$ are given by

$$
\gamma_{x}^{\delta}=\mathrm{id} \wedge \gamma_{x} \delta, \text { for } x \in E
$$

This construction is illustrated in Fig. 8. Although the previous exposition is limited to the
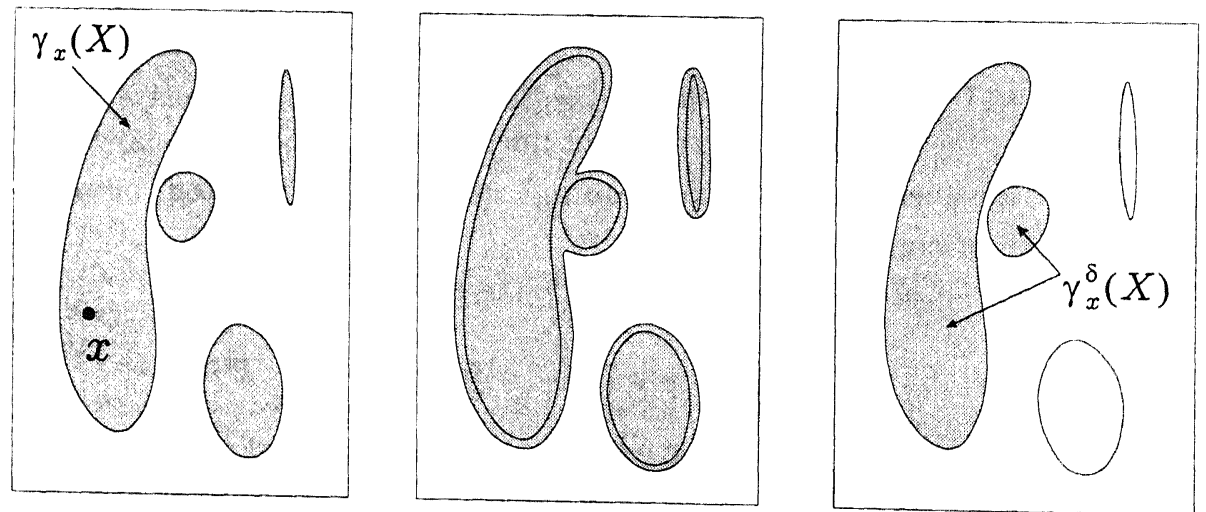

Figure 8 From left to right: a set $X$ and the grain $\gamma_{x}(X)$, the dilation $\delta(X)$, and the grain $\gamma_{x}^{\delta}(X)$.

complete lattice $\mathcal{P}(E)$, the concept of connectivity class can be extended to the case of an arbitrary complete lattice $\mathcal{L}$. For more information, refer to [26] or [27]. 


\subsection{Connected operators}

Classical morphological operators, like the ones discussed in Section 3, are compositions of one or more "local" operators. Here, "local" means that the output of an operator at a particular point depends only on the input values in a small neighborhood around this point. In contrast, the connected operators to be discussed in this section do not change the value of individual pixels but only the values of connected regions with constant grey-level, the so-called flat zones. In this section, we restrict our exposition to the binary case. The flat zones are therefore the connected components of the foreground and the background. For the grey-level case, the reader is referred to [26].

Before we proceed with a formal definition of a connected operator, we need the concept of a partition, which subdivides a given space $E$ into disjoint zones. Given a space $E$, a function $P$ : $E \rightarrow \mathcal{P}(E)$ is called a partition of $E$ if: (i) $x \in P(x)$, for every $x \in E$, and (ii) $P(x)=P(y)$, or $P(x) \cap P(y)=\emptyset$, for every $x, y \in E$. The set $P(x)$ is called the $z$ one of partition $P$ that contains point $x$. If $E$ is furnished with a connectivity $\mathcal{C}$, and if $P(x) \in \mathcal{C}$, for every $x \in E$, then we say that $P$ is a connected partition. Given two partitions $P$ and $P^{\prime}$ of a space $E$, we say that $P$ is coarser than $P^{\prime}$ (or that $P^{\prime}$ is finer than $P$ ) if $P^{\prime}(x) \subseteq P(x)$, for every $x \in E$.

Every subset $X$ of $E$ can be associated with a connected partition $P_{X}$ the zones of which are the grains of $X$ and $X^{c}$. In this case, we have that

$$
P_{X}(h)=\left\{\begin{array}{ll}
\gamma_{h}(X), & \text { if } h \in X \\
\gamma_{h}\left(X^{c}\right), & \text { if } h \notin X
\end{array} .\right.
$$

Notice that this connected partition directly depends on the choice of connectivity.

An operator $\psi$ on $\mathcal{P}(E)$ is called a connected operator if the partition $P_{\psi(X)}$ is coarser than the partition $P_{X}$, for every subset $X \subseteq E$. Notice that a connected operator acts on the zones of an image $X$, by preserving certain zones and by changing others so that the partition of the input image is coarsened. It can be shown that an operator $\psi$ is connected if and only if the symmetric difference $(X \backslash \psi(X)) \cup(\psi(X) \backslash X)$ consists only of grains of $X$ and $X^{c}$, for every $X \subseteq E[13,28]$.

An important example of a connected operator is given by

$$
\check{\alpha}(X)=\rho(\alpha(X) \mid X), \text { for } X \in E,
$$

where

$$
\rho(Y \mid X)=\bigcup_{y \in Y} \gamma_{y}(X), \text { for } X, Y \in E
$$

is the so-called reconstruction of $Y$ in $X[10]$, and $\alpha$ is a given opening. The operator $\check{\alpha}$ is an opening on $\mathcal{P}(E)$, known as opening by reconstruction. In fact, every connected opening (i.e., an opening that is also a connected operator) is necessarily of the form (24); see [13]. Fig. 9 depicts an example of such an operator, when $\alpha(X)=X \circ A$, with $A$ being a disk structuring element. Notice that, in this case, operator $\check{\alpha}$ reconstructs only the connected components of $X$ which are "marked" by the opening $X \circ A$. 


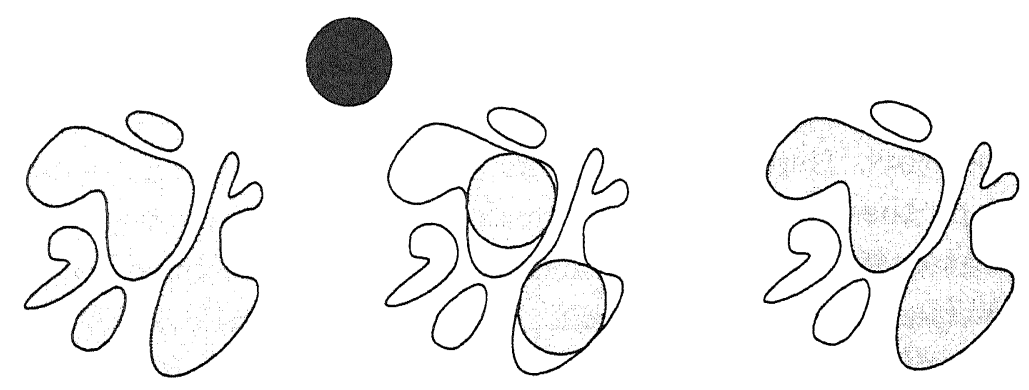

Figure 9 Opening by reconstruction. From left to right: $X, \alpha(X)$, and $\breve{\alpha}(X) . \alpha(X)$ is an opening by a disk structuring element (in black).

\subsection{Grain operators}

Given a connectivity $\mathcal{C}$ on $E$, by a grain criterion we mean a mapping $u: \mathcal{C} \rightarrow\{0,1\}$. Given two grain criteria $u$ and $v$, we define a grain operator $\psi_{u, v}(X)$ by $[2,13]$

$$
\psi_{u, v}(X)=\left(\bigcup\left\{\gamma_{x}(X) \mid u\left(\gamma_{x}(X)\right)=1\right\}\right) \bigcup\left(\bigcup\left\{\gamma_{x}\left(X^{c}\right) \mid v\left(\gamma_{x}\left(X^{c}\right)\right)=0\right\}\right) .
$$

Clearly, this operator leaves unchanged foreground grains $\gamma_{x}(X)$ that satisfy criterion $u\left(\gamma_{x}(X)\right)=$ 1 and background grains $\gamma_{x}\left(X^{c}\right)$ that satisfy criterion $v\left(\gamma_{x}\left(X^{c}\right)\right)=1$, and flips the other grains from foreground to background and vice versa. An example, illustrating this concept, is depicted in Fig. 10.
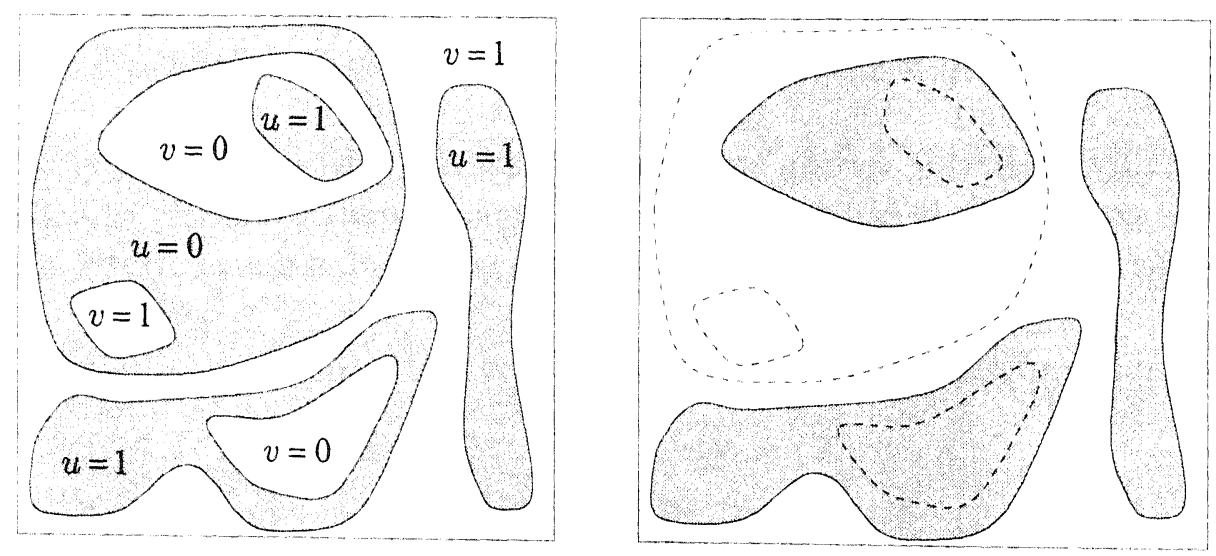

Figure 10 A binary image $X$ (left) and its transform $\psi_{u, v}(X)$ (right). In every foreground (resp. background) grain of $X$ it is indicated whether the grain criterion $u$ (resp. $v$ ) equals 0 or 1.

It can be shown that a connected operator $\psi: \mathcal{P}(E) \rightarrow \mathcal{P}(E)$ is a grain operator if and only if, for every $h \in E$ and $X, Y \subseteq E$ such that $X(h)=Y(h)$ and $P_{X}(h)=P_{Y}(h)$, we have that $\psi(X)(h)=\psi(Y)(h)[13]$. This property means that $\psi$ is a grain operator if the value $\psi(X)(h)$ is completely determined by the value $X(h)$ and the zone $P_{X}(h)$. 
An example of a grain operator is obtained by considering the grain criteria

$$
u(C)= \begin{cases}1, & \text { if }|C| \geq t \\ 0, & \text { otherwise }\end{cases}
$$

for some nonnegative threshold $t$, where $|C|$ denotes the area of set $C$, and $v(C)=1$, for every $C \in \mathcal{C}$. The resulting operator is an opening, known as an area opening. This operator deletes from a set $X$ all grains with area less than $t$. Another example of a grain operator is the opening by reconstruction, given by (24).

\section{Conclusion}

In this paper, we have provided a brief introduction to a powerful tool for shape analysis known as mathematical morphology. We have limited our presentation to various theoretical aspects, by focusing on the theory of morphological operators on complete lattices. We hope that we have succeeded in giving the reader a first impression of the underlying algebraic principles of mathematical morphology, and the fact that this tool benefits from the power of a complete lattice theoretic framework.

\section{References}

[1] Bloch, I., and Maitre, H.: "Fuzzy mathematical morphologies: A comparative study," Pattern Recognition, 28, 1995, 1341-1387.

[2] Crespo, J., and Schafer, R. W.: "Locality and adjacency stability constraints for morphological connected operators," Journal of Mathematical Imaging and Vision, 7, 1997, 85-102.

[3] Dougherty, E. R.: An Introduction to Morphological Image Processing, Bellingham, Washington: SPIE Optical Engineering Press, 1992.

[4] Dougherty, E. R. (ed.): Mathematical Morphology in Image Processing, New York: Marcel Dekker, 1993.

[5] Dougherty, E. R., and Astola, J. T. (eds.): Nonlinear Filters for Image Processing, Bellingham, Washington: SPIE Optical Engineering Press, 1999.

[6] Giardina, C. R., and Dougherty, E. R.: Morphological Methods in Image and Signal Processing, Englewood Cliffs, New Jersey: Prentice-Hall, 1988.

[7] Goutsias, J., Heijmans, H. J. A. M., and Sivakumar, K.: "Morphological operators for image sequences," Computer Vision and Image Understanding, 62, 1995, 326-346.

[8] Haralick, R. M., Sternberg, S. R., and Zhuang, X.: "Image analysis using mathematical morphology," IEEE Transactions on Pattern Analysis and Machine Intelligence, 9, 1987, $532-550$.

[9] Heijmans, H. J. A. M.: "Theoretical aspects of gray-level morphology," IEEE Transactions on Pattern Analysis and Machine Intelligence, 13, 1991, 568-582. 
[10] Heijmans, H. J. A. M.: Morphological Image Operators, Boston, Massachusetts: Academic Press, 1994.

[11] Heijmans, H. J. A. M.: "Self-dual morphological operators and filters," Journal of Mathematical Imaging and Vision, 6, 1996, 15-36.

[12] Heijmans, H. J. A. M.: "Easy recipes for morphological filters." E. R. Dougherty and J. Astola (eds.), Nonlinear Filters for Image Processing, SPIE Optical Engineering Press, Bellingham, Washington, 1999.

[13] Heijmans, H. J. A. M.: "Connected morphological operators for binary images," Computer Vision and Image Understanding, 73, 1999, 99-120.

[14] Heijmans, H. J. A. M., and Ronse, C.: "The algebraic basis of mathematical morphology - Part I: Dilations and erosions," Computer Vision, Graphics and Image Processing, 50, 1990, 245-295.

[15] Heijmans, H. J. A. M., and Serra, J.: "Convergence, continuity, and iteration in mathematical morphology," Journal of Visual Communication and Image Representation, 3, 1992, 84-102.

[16] Maragos, P.: "A representation theory for morphological image and signal processing," IEEE Transactions on Pattern Analysis and Machine Intelligence, 11, 1989, 586-599.

[17] Maragos, P.: "Pattern spectrum and multiscale shape representation," IEEE Transactions on Pattern Analysis and Machine Intelligence, 11, 1989, 701-716.

[18] Maragos, P., and Schafer, R. W.: "Morphological filters - Part I: Their set-theoretic analysis and relations to linear shift-invariant filters," IEEE Transactions on Acoustics, Speech and Signal Processing, 35, 1987, 1153-1169.

[19] Maragos, P., and Schafer, R. W.: "Morphological filters - Part II: Their relations to median, order-statistic, and stack filters," IEEE Transactions on Acoustics, Speech and Signal Processing, 35, 1987, 1170-1184.

[20] Matheron, G.: Random Sets and Integral Geometry, New York: John Wiley \& Sons, 1975.

[21] Popov, A. T.: "Convexity indicators based on fuzzy morphology," Pattern Recognition Letters, 18, 1997, 259-267.

[22] Ronse, C.: "Why mathematical morphology needs complete lattices," Signal Processing, 21, 1990, 129-154.

[23] Schmitt, M., and Mattioli, J.: Morphologie Mathématique, Paris, France: Masson, 1994.

[24] Serra, J.: Image Analysis and Mathematical Morphology, London, England: Academic Press, 1982.

[25] Serra, J. (ed.): Image Analysis and Mathematical Morphology. II: Theoretical Advances, London, England: Academic Press, 1988.

[26] Serra, J.: "Connectivity on complete lattices," Journal of Mathematical Imaging and Vision, 9, 1998, 231-251.

[27] Serra, J.: "Connectivity for sets and functions," Fundamenta Informaticae, 27, 2000.

[28] Serra, J., and Salembier, P.: "Connected operators and pyramids," SPIE Proceedings on Image Algebra and Morphological Image Processing IV, San Diego, 2030, 1993, 65-76. 
[29] Sinha, D., and Dougherty, E. R.: "Fuzzy mathematical morphology," Journal of Visual Communication and Image Representation, 3, 1992, 286-302.

[30] Sinha, D., Sinha, P., Dougherty, E. R., and Batman, S.: "Design and analysis of fuzzy morphological algorithms for image processing," IEEE Transactions on Fuzzy Systems, 5, 1997, 570-584.

[31] Soille, P.: Morphological Image Analysis, Berlin, Germany: Springer-Verlag, 1999.

[32] Sternberg, S. R.: "Grayscale morphology," Computer Vision, Graphics and Image Processing, 35, 1986, 333-355.

[33] Vincent, L.: "Morphological algorithms." E. R. Dougherty (ed.), Mathematical Morphology in Image Processing, Marcel Dekker, New York, 1993, 255-288.

[34] Vincent, L., and Soille, P.: "Watersheds in digital spaces: An efficient algorithm based on immersion simulations," IEEE Transactions on Pattern Analysis and Machine Intelligence, 13, 1991, 583-598.

[35] Wendt, P. D., Coyle, E. J., and Callagher, N. C.: "Stack filters," IEEE Transactions on Acoustics, Speech and Signal Processing, 34, 1986, 898-911. 